\title{
Forschungsinstitut
}

\section{Freie Berufe-}

Europäische Entwicklungen

\author{
Harald Herrmann
}

FFB-Diskussionspapier Nr. 72

Mai 2007

\section{$\notin$ \\ LEUPHANA \\ UNIVERSITÄT LÜNEBURG}

\section{Fakultät II - Wirtschafts-, Verhaltens- und Rechtswissenschaften}

Postanschrift:

Forschungsinstitut Freie Berufe

Postfach 2440

21314 Lüneburg ffb@uni-lueneburg.de

http://ffb.uni-lueneburg.de

Tel: +49 4131 677-2051

Fax: +49 4131 677-2059 


\section{Freie Berufe - \\ Europäische Entwicklungen}

\section{Harald Herrmann ${ }^{1}$}

FFB-Diskussionspapier Nr. 72

Mai 2007

ISSN 0942-2595

1 Prof. Dr. Harald Herrmann, Foprschungsinstitut Freie Berufe (FFB), Friedrich-Alexander-Universität Erlangen-Nürnberg, Lange Gasse 20, 90403 Nürnberg, Tel.: 0911/5302-267, Fax: 0911/5302-177, e-mail: Herrmann@wiso.uni-erlangen.de 


\title{
Freie Berufe - Europäische Entwicklungen \\ Harald Herrmann \\ FFB-Diskussionspapier Nr. 72, Mai 2007, ISSN 0942-2595
}

\section{Zusammenfassung}

Der Vorschlag der EU-Kommission zur Deregulierung Freier Berufe von 2003 sieht vor, reglementierte Berufe in einer Kategorie zusammenzufassen. Dadurch sollen Berufe verschiedenster Art mit Freiberufen regulatorisch zusammengefügt werden, die weder die bisher üblichen Voraussetzungen der Unabhängigkeit noch solche der Eigenständigkeit und wissenschaftlichen Qualifikation erfüllen.

Die Wiener Studie über die Freiberufsrechte in Europa von 2002 von Ogus et al. hat bestätigt, dass nicht nur erhebliche Unterschiede der Regulierungsdichte bestehen, sondern dass es auch Deregulierungstrends gibt, wie sie in der Lüneburger Studie von 1996 bereits großenteils übereinstimmend nachgewiesen wurden. Neuere Ergebnisse dieser Trendforschung sollten im Zusammenhang aktueller Theorien des sogenannten Systemwettbewerbs eingebracht werden.

Der frühere Kommissar Monti hat eine Initiative zur Bekämpfung kartellrechtswidriger Regulierungen Freier Berufe eingeleitet. Sie wird fortgesetzt, aber dadurch relativiert, dass moderne Compliance-Strategien berücksichtigt werden.

JEL: J20, J23, J51

Schlagwörter: Freie Berufe, Deregulierung, Freiberufsrechte in Europa, Wiener Studie, Systemwettbewerb

\begin{abstract}
The suggestion of the EU Commission concerning the deregulation of liberal professions from 2003 plans to regulated occupations into one category. Various occupations, which have not fulfilled the conditions of independence and scientific qualification, yet, should be thereby being joined with the liberal professions.

The Viennese study about liberal profession rights in Europe of 2002 by Ogus et al. has confirmed that not only considerable differences of the regulation density exist, but that there are also deregulation-trends as they were already proved in the Lüneburg-study from 1996 with correspondence. Newer results of this trend research should be introduced in the connection of topical theories of the so-called system competition.

The former commissioner Monti has initiated an initiative for the abatement of cartel-illegal regulations of free professions. It is continued, but is weakened by the fact that modern compliance strategies are taken into consideration.
\end{abstract}

JEL: J20, J23, J51

Keywords: Liberal Professions, Deregulation, liberal occupation rights in Europe, Viennes study, system competition 


\section{Freie Berufe - Europäische Entwicklungen}

\section{Harald Herrmann}

\section{Freie Berufe - Europäische Entwicklungen}

Die europäische Entwicklung der Freiberufsrechte hat seit langem zwei Seiten: eine transnationale, direkt vom Europarecht ausgehende und eine internationale, die im Wettbewerb auf den internationalen Freiberufsmärkten wurzelt. Zu beiden Entwicklungssträngen sind in den letzten zwei Jahren erhebliche Anstöße und Vorgänge zu verzeichnen, die in der Tabelle 1 veranschaulicht werden.

\section{Tabelle 1: $\quad$ Die zwei Seiten europäischer Entwicklung der Freiberufsrechte}

\begin{tabular}{|c|c|c|c|}
\hline & Rechtsquelle & Grund & Beispiele \\
\hline transnational & EGV/Richtl./VO & $\begin{array}{l}\text { non-tarif barriers of } \\
\text { entry }\end{array}$ & $\begin{array}{l}\text { - Kartellverbot von } \\
\bullet \quad \text { Berufsordnungen } \\
\bullet \quad \text { (Werbg. um Praxis) } \\
\text { - Richtl. Anerk. } \\
\text { Berufsqualifikationen }\end{array}$ \\
\hline international & $\begin{array}{l}\text { internat. } \\
\text { Rechtsvergleich }\end{array}$ & Systemwettbewerb & $\begin{array}{l}\text { - Trend „weiche“ } \\
\text { Kammerformen } \\
\text { - Clementi-Reformen }\end{array}$ \\
\hline
\end{tabular}

Quelle: Eigene Darstellung

Bei den transnationalen Vorgängen scheint mit am wichtigsten der Vorstoß des früheren Wettbewerbskommissars Monti, mit der Keule des Kartellrechts gegen wettbewerbsbeschränkende Berufsordnungen vorzugehen. Die Initiative beruht auf dem primärrechtlichen Kartellverbot des Art. 81 EGV und birgt die Gefahr in sich, dass bei Nichtigkeit der verbotenen Regeln erhebliche Lücken in die Freiberufsrechte gerissen werden. Dies wird in diesem Beitrag anhand des in zahlreichen deutschen Berufsordnungen geregelten Verbots der Werbung um Praxis erläutert. Dazu wird ein Lösungsansatz vorgestellt, der mit weniger drastischen Folgen für die berufsrechtliche Selbstverwaltung verbunden ist.

Es folgt eine kurze Vorstellung des Richtlinienvorschlags zur Anerkennung von Berufsqualifikationen in so genannten reglementierten Berufen. Die Kommission übergeht hier den vermeintlich rein deutschrechtlichen Begriff des Freien Berufs und muss hieran, wie mir mit vielen anderen scheint, nach Kräften gehindert werden. Hierfür werden Gründe aufgezeigt und es wird erläutert, welches Vorgehen in dieser Hinsicht aussichtsreich erscheint.

Letztlich folgt ein rechtsvergleichender Blick auf die von vielen als revolutionär empfundenen Clementi-Reformen in England. Mit ihnen stehen die Freiberufskammern vor einer Entmachtung im Hinblick auf alle Aufgaben der so genannten Selbstverwaltung und könnten zu reinen Interessenverbänden verkümmern. Da die Entwicklung bereits nach Frankreich und in 
die Niederlande ausgestrahlt hat, mahnt der Präsident des europäischen Anwaltsverbandes Hellwig eindringlich zu rechtzeitigen Entschlackungen des deutschen Kammerrechts. Auch dies wird am Beispiel der Werbung um Praxis veranschaulicht.

\section{$2 \quad$ EU-Kartellrechtscompliance für Berufsordnungen mit Werbeverboten}

Dieser Abschnitt widmet sich den Fragen zum europarechtlichen Kartellverbot. Manche kammerrechtliche Werbeverbote erfüllen den Kartelltatbestand des Art. 81 Abs. 1 EGV und sind dennoch nach der vom EuGH in der Wouters-Entscheidung ${ }^{1}$ von 2002 anerkannten rule of reason gerechtfertigt. Folgende Hauptaspekte werden nochmals zusammengefasst.

\subsection{Werbung um Praxis und Ambulance Chasing}

Art. 81 Abs. 1 EGV verbietet „....alle Vereinbarungen zwischen Unternehmen, Beschlüsse von Unternehmensvereinigungen und aufeinander abgestimmte Verhaltensweisen, welche den Handel zwischen Mitgliedstaaten zu beeinträchtigen geeignet sind und eine Verhinderung, Einschränkung oder Verfälschung des Wettbewerbs innerhalb des Gemeinsamen Marktes bezwecken oder bewirken...“

In den meisten Freiberufsrechten haben sich heute Werbevorschriften durchgesetzt, die unter dem Begriff der Informationswerbung zusammengefasst werden. Damit wird meist das Verbot der Werbung um Praxis verbunden. Beispiele sind die §§ 43b BRAO, 57a StBerG, 52 S.2 WPO, in denen die Bestimmung sogar als förmliches Gesetz geregelt ist. Darin liegt zunächst noch keine Kartellverbotsverletzung, da die gesetzliche Anordnung kein Verbandsbeschluss i.

S. Art. 81 EGV ist.

Doch kommt nun alles darauf an, was man unter „um Praxis“ versteht. Während die BRAK darauf verzichtet hat, dazu satzungsrechtliche Einzelheiten zu regeln, haben die Bundessteuerberaterkammer und die Wirtschafsprüferkammer folgende Regelungen getroffen: $\S 10$ Abs.3 BOStB ${ }^{2}$ verbietet Werbung, „....so weit sie auf die Erteilung eines Auftrags im Einzelfall gerichtet ist“ und „,...der Umworbene in einem konkreten Einzelfall der Beratung oder der Vertretung bedarf und der Werbende dies in Kenntnis der Umstände zum Anlass für seine Werbung nimmt“. Ähnlich ist $\S 35$ BO/WP/vBP gefasst ${ }^{3}$, wonach ebenfalls die Ausrichtung auf den Einzelfall unzulässig ist, „....wenn hierzu eine Aufforderung des möglichen Auftraggebers vorliegt“.

In der neueren Literatur wird gegen diese weite Fassung des Werbeverbots eingewendet, dass in der bloßen Ausrichtung auf Einzelpersonen keine unsachliche Beeinflussung liegt, wenn die Werbung nicht so adressiert ist, dass besondere Notlagen oder Schamgefühle des Nachfragers ausgenutzt werden. ${ }^{4}$ Beispiele für solches Verhalten sind das Ansprechen am Unfall-

1 Rs.C-309/99, Wouters, Savelbergh/Algemene Raad van de Nederlandse Orde van Adocaten, NJW 2002, 877.

$2 \S 10$ Berufsordnung der Bundessteuerberaterkammer vom 2.6.1997 in der Version vom 24.10.2001, Deutsches Steuerrecht 12/2002, 518 s., Wiederabdruck in. Kleine-Cosack, 2004, 301 ff.

$3 \S 35$ Berufsordnung der Wirtschaftsprüfer/vereidigte Buchprüfer vom 11.6.1996 i.d.F. v. 11.3.2002, BAnz. 2002, at 789; Wiederabdruck ebd., $303 \mathrm{ff}$.

4 Hellwig 2005, 1217, 1219; Kleine-Cosack 2004, Rdn. 342 ff. m.w.Nachw. 
ort, beim Verlassen von Amträumen, in denen dem Betroffenen Strafen oder Geldbußen auferlegt wurden, und dergleichen. Man spricht insoweit von ambulance chasing. Erst bei derart sachwidriger Beeinflussung dürfe Werbung um Praxis verboten sein.

Zunächst ergibt sich aus der Gegenüberstellung, dass die genannten Berufsordnungen über den berechtigten Inhalt des Verbots der Werbung um Praxis hinausgehen und deshalb als vom Gesetz losgelöste Verbandsbeschlüsse i.S. des Art. 81 EGV anzusehen sind. Zwar mag der Wortlaut der Gesetzesbestimmungen jedweden Individualkontakt als Praxiswerbung mit umfassen, doch scheint insoweit eine enge Auslegung geboten. Denn wenn man die Freien Berufe dem Wettbewerb öffnet, dann muss auch hier der allgemeine Grundsatz gelten, wonach Werbung nicht als solche, sondern nur bei unsachlicher oder unfairer Beeinflussung verboten ist.

\subsection{Geltungsausnahmen versus Rule of Reason}

Für die weitere Beurteilung kommt es darauf an, ob Kammerrechte generell von der Geltung des Kartellverbots ausgenommen sein sollen, oder ob dies nur bei Vorliegen besonderer Rechtfertigungsgründe der Fall sein soll. Hierzu hat der EuGH in der Arduino-Entscheidung ${ }^{5}$ von 2002 geurteilt, dass eine Geltungsausnahme nur dann in Betracht kommt, wenn es sich um einen Verbandsbeschluss handelt, der als solcher nicht wirksam wird, sondern einem so genannten Letztentscheid einer staatlichen Stelle unterliegt. Entgegen z.T. vertretener Ansicht reicht hierfür die staatliche Kammeraufsicht deutschen Rechts nicht aus.

Es bleibt also gar nichts anderes übrig, als nach besonderen Rechtfertigungsgründen des Einzelfalles zu suchen. Dafür sieht an sich Art. 81 Abs. 3 EGV eine ganze Reihe von so genannten Freistellungsmerkmalen vor. Doch sind diese allesamt auf ökonomische Effizienzvorteile von Rationalisierungskartellen, Spezialisierungsabreden und dergleichen bezogen, so dass eine Freistellung für Wettbewerbsbeschränkungen durch Berufskammern nicht in Betracht kommt. Doch hat der EuGH in einer weiteren neuen Entscheidung im Falle Wouters ${ }^{6}$ betont, dass der Freistellungstatbestand des Art. 81 Abs. 3 EGV keine abschließende Regelung enthält, sondern daneben noch eine allgemeine Rechtfertigung wegen überwiegender öffentlicher Interessen möglich ist. Da eine derartige Güterabwägung im US-amerikanischen Kartellrecht seit mehr als 100 Jahren als rule of reason zugelassen wird ${ }^{7}$, scheint die Ansicht des EuGH im Falle Wouters zumindest rechtsvergleichend gut bestätigt.

Daraus folgt, dass die Abwägung öffentlicher Interessen an der Wettbewerbsbeschränkung mit den durch diese bedingten Wettbewerbsnachteilen einem strengen Übermaßverbot zu folgen hat, d.h. das betreffende Werbeverbot darf niemals weiter gehen, als es unbedingt erforderlich ist, um unsachliche Beeinflussungen der Nachfrager zu vermeiden. Wie gezeigt, ist dies nur beim Verbot des ambulance chasing, nicht aber bei einem weiter gefassten Verbot der Werbung um Praxis der Fall. Die zitierten Berufsordnungen der Bundessteuerberaterkammer sowie der Wirtschaftsprüfer und vereidigten Buchprüfer sind also als Verletzungen des Art. 81 EGV anzusehen, eine Ansicht, die in den neueren Veröffentlichungen zum Thema zumindest in abstrakter Form längst konsentiert erscheint. Dass gleichwohl, soweit ersicht-

\footnotetext{
EuGH NJW 2002, 882, Rs. C-35/99 Manuele Arduino

A.a.O., Fn. 2.

Grdl. Standard Oil ,Co. of New Jersey v. U.S., 221 U.S.. 1 (1911); zur weiteren Entwicklung Ackermann, Art. 85 Abs. 1 EGV und die Rule of Reason, 1997, $11 \mathrm{ff.}$
} 
lich, keine Novellierung in den betroffenen Kammern in Angriff genommen worden ist, ist nicht unverständlich, aber Grund genug, die kartellrechtlichen Bedenken an dieser Stelle noch einmal deutlich zu artikulieren.

\subsection{Compliance durch Verpflichtungszusagen oder Governance-Kodex}

Betrachtet man des Weiteren, welche Sanktionsmöglichkeiten drohen und wie die Wettbewerbsdirektion der EU mit diesem Phänomen praktisch umgehen sollte. Vor ungefähr Jahresfrist hat der Autor in einem Forschergremium beim ehemaligen Direktionsleiter Ehlermann vorgetragen, dass es keinen Sinn macht, in diesen und zahlreichen ähnlichen Fällen schlicht Verbotsverfügungen gegen die betroffenen Kammern zu erlassen (Herrmann 2004). Denn auf diesem Wege würde man dem Verbraucher, dessen Schutz das Kartellverbot letztlich bezweckt, Steine statt Brot geben. Es wäre gar kein Werbeverbot mehr rechtsgültig, obgleich man das des ambulance chasing, das nach der rule of reason auch eindeutig gerechtfertigt wäre, dringend benötigt.

Notwendig ist also nicht der Erlass rigider Verbotsverfügungen, sondern die Anregung wettbewerbsrechtskonformen Verhaltens der Kammern und Verbände Freier Berufe. Schließlich kann die Wettbewerbsdirektion nicht eine eigene Berufsregelung treffen, nach der nicht mehr die weite Fassung des Verbots der Werbung um Praxis, stattdessen aber die des ambulance chasing gelten soll. Die betroffenen Verbände müssen selbst tätig werden, und sie müssen dies in der Überzeugung tun, dass soviel Wettbewerb wie möglich und so viel Werbeverbot wie nötig gewollt ist.

Um diese Aufgabe der Kartellbehörde richtig anzugehen, muss man die neueren Erkenntnisse der Compliance-Forschung berücksichtigen. Darunter versteht man eine bahnbrechende neuartige Form der ökonomischen Rechtsanalyse (vgl. nur Dreher 2004, 1 ff.; zum Gesellschaftsrecht s. Christine Parker 2001), die nicht mehr so sehr auf die gesamtwirtschaftliche Wohlfahrtsoptimierung, sondern darauf abstellt, welche einzelwirtschaftlichen Anreize gegeben werden können, um Betriebe oder Verbände zur ökonomisch effektiven Befolgung von Recht zu veranlassen. Der methodische Hintergrund dieser Rechtsökonomik kann hier natürlich nicht weiter beleuchtet werden (s. Herrmann 2004a, 58 ff.). Doch lässt sich diese neue ökonomisch-rechtsanalytische Sicht auch für die freiberufsrechtliche Problematik, wie folgt, nutzbar machen:

Man kann entweder abwarten, bis die Kommission ein Verfahren gegenüber einem Berufsverband eröffnet, einen Verstoß gegen Art. 81 EGV festgestellt hat und ihr Nichteinschreiten gegen Verpflichtungszusage erklärt. Oder es wird von vornherein ein Kartellrechtsverfahren vermieden, indem die Kommission eine Art Kodex verkündet, der dem der aktienrechtlichen corporate governance codes in Europa strukturell entspricht. Zunächst sei das geregelte Verfahren vorgestellt.

Art. 9 Abs. 1 VO 1/2003 gibt der Kommission die relativ neuartige Option, bei Feststellung einer Kartellverbotsverletzung von einer Verbotsverfügung abzusehen und stattdessen eine Nichteinschreitenserklärung abzugeben, wenn der Verband eine so genannte Verpflichtungszusage erklärt. Diese muss besagen, dass anstelle des beanstandeten Werbeverbots eine Regelung getroffen wird, die mit dem Übermaßverbot der rule of reason in Einklang steht, also in unserem Fall auf das Verbot des ambulance chasing reduziert ist. Im Vorgriff auf eine solche Lösung kann die Behörde selbstverständlich signalisieren, dass sie eine solche Regelung so- 
wohl für notwendig als auch für ausreichend hält. Handelt es sich um ein Musterverfahren, so kann man sich auch von dieser Vorgehensweise eine gewisse Breitenwirkung versprechen.

Vorzugswürdig scheint aber - gerade im Hinblick auf die Breitenwirkung - ein professional governance code, der dem bekannten aktienrechtlichen Corporate-governance-Kodex der deutschen Regierungskommission ${ }^{8}$ und zahlreicher entsprechender Organisationen in den Gemeinschaftsländern der Struktur nach entspricht. Dafür ist der Begriff der professional governance international verbreitet. Inhaltlich geht es um Empfehlungen guter Berufsverbandsaufsicht, die nicht von der EU-Kommission selbst, sondern von einem von ihr beauftragten Expertengremium formuliert werden. Natürlich wären hier viel weitergehende Inhalte abzudecken, als nur die bereits erörterten europarechtskonformen Werbeverbote. Aber diese wären ein wichtiger Bestandteil; und es soll hier erwähnt werden, dass in der Kommission derzeit darüber nachgedacht wird, einen unverbindlichen Compliance-Kodex dieser Art auszuarbeiten. Man kann nur hoffen, dass dieser echte Compliance-Elemente beinhalten wird, d.h. das Grundprinzip der Professionalisierung und der damit notwendig verbundenen Selbstverwaltung berücksichtigt. Leider gibt es politische Zweifel daran, die im Folgenden deutlich werden.

\section{$3 \quad$ EU-Richtlinie Berufsqualifikationen}

\subsection{Verfahrensstand und Eckdaten}

Folgend soll es um den sekundärrechtlichen Europarechtsteil, den Richtlinienentwurf über die Anerkennung von Berufsqualifikationen (Kommissionsvorschlag 2002) gehen. ${ }^{9}$ Der erste Vorschlag der Kommission datiert von 2002 und wurde nach dem so genannten Mitentscheidungsverfahren i.S. Art. 251 EGV dem Europäischen Parlament zugeleitet. Im Gegensatz zu dem durch die Einheitliche Europäische Akte eingeführten Verfahren der institutionellen Zusammenarbeit i.S. Art. 252 EGV kann das Parlament durch Ablehnung mit absoluter Mehrheit seiner Mitglieder das endgültige Scheitern des Vorhabens herbeiführen (Art. 251 Abs. 2 lit.b EGV).

Zunächst wurde der Kommissionsvorschlag 2002 vom Parlament in einer Entschließung vom 11.2.2004 in 125 Punkten abgeändert. Die Kommission hatte nun nach Art. 250 Abs. 2 EGV die Kompetenz, einen geänderten Richtlinienvorschlag zu unterbreiten, und hat hiervon am 20.4.2004 Gebrauch gemacht. ${ }^{10}$ Mehr als die Hälfte der Parlamentsänderungen, nämlich 70 Punkte, werden von der Kommission nicht übernommen, so dass das Zustandekommen der Richtlinie derzeit nicht mit Sicherheit vorhergesagt werden kann. Beim Rat, der schließlich auf Ablehnungsbeschluss des Parlaments nur einstimmig entscheiden kann, wurde eine konsolidierte Fassung erarbeitet. Diese wird zurzeit in einer Ratsarbeitsgruppe „Niederlassungsrecht und Dienstleistungen“ diskutiert (Mann 2004, 615). Lehnt das Parlament die Neufassung durch die Kommission nach Einschaltung des Vermittlungsausschusses mit absoluter Mehrheit ab, so ist sie gescheitert (Art. 251 Abs. 5 EGV).

8 Downloading unter www.corporate-governance-code.de; dazu Ringlieb, Kremer, Lutter, v. Werder 2003.

9 Vorschlag für eine Richtlinie des europäischen Parlaments und des Rates über die Anerkennung von Berufsqualifikationen v. 7.3.2002, KOM (2002) 119 endg.; dazu Mann 2004, 615 ff.

10 Kommission, Geänderter Vorschlag für eine Richtlinie des Europäischen Parlaments und des Rates über die Anerkennung von Berufsqualifikationen v. 20.4.2004, KOMM (2004) 317 endg. 
Zur Veranschaulichung der Besonderheiten des Mitentscheidungsverfahrens dient die Abbildung 1.

\section{Abbildung 1: Besonderheiten des Mitentscheidungsverfahrens}

\section{Zusammenarbeit, Art. 252 EGV}

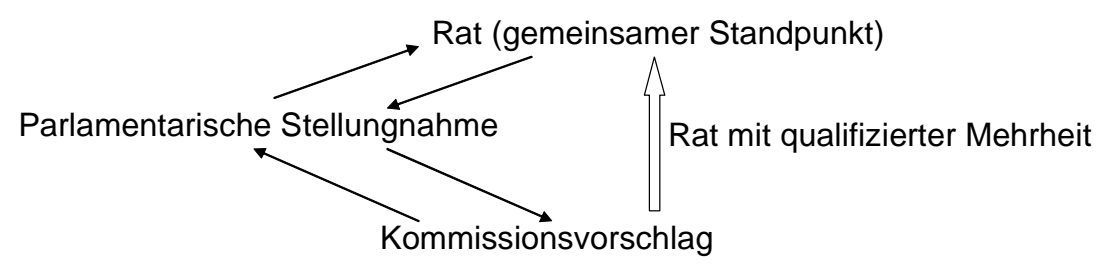

\section{Mitentscheidung, Art. 251 EGV}

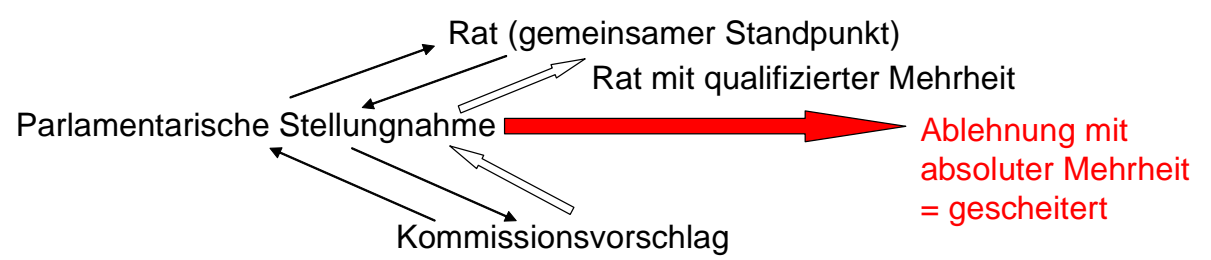

Quelle: Eigene Darstellung

Zentraler Punkt des ursprünglichen Kommissionsvorschlags und des Änderungsvorschlags ist die Zusammenfassung der Anerkennungsregeln für Freie Berufe und andere so genannte reglementierte Berufe. Freiberufe rangieren zwar auf der obersten von 5 Anerkennungsstufen, dem Hochschuldiplom mit 4-jährigem Studiengang (Art. 11 Abs. 5 Kommissionsvorschlag 2002), doch kommt es weder hier noch auf der nächst niedrigeren Stufe, dem Diplom mittlerer Ausbildungsgang, auf die typusbildenden Freiberufsmerkmale der persönlich, eigenverantwortlich und fachlich unabhängigen, geistig-ideellen Leistung an. Zudem rangieren zwar die Fachhochschuldiplome mittlerer Ausbildungsgang mit 3-jähriger Zeitdauer auf der 4. Stufe, doch ist bei Unterschreitung des Migranten um 1 Stufe grundsätzlich die Anerkennung im Gastland vorgesehen (Art. 13 Abs. 1b Kommissionsvorschlag 2002).

\subsection{Der Freiberufsansatz des EU-Parlaments 2004}

Unterscheitungen der Qualifikationsanforderungen in Zielländern können demzufolge zu Deprofessionalisierungen führen, die mit der weltweit nachgewiesenen Entwicklung Freier Berufe (vgl. nur Conze/ Kocka 1992; Herrmann 1996, 56 ff. m.w.Nachw) ganz und gar unvereinbar wären. Aus der Sicht der freiberuflichen Professionalisierung folgt nach wie vor erhebliche Kritik an diesem Grundanliegen des Kommissionsvorschlags, die Stufe unterhalb der Freiberuflichkeit zur Anerkennung im Gastland zu bringen, wenn es denn im Herkunftsland so gehandhabt wird. Die Sorge um die Zerstörung Freier Berufe ist dem Parlament im Anhörungsverfahren sowohl vom DAV, nach dem Rat des Autors (Herrmann 2003), als auch von verschiedenen anderen Stellen, insbesondere dem BfB (2002), nachhaltig vorgetragen worden. Die Kritik des DAV sei folgend noch einmal zusammengefasst: 
85\% der im DAV vertretenen Astrologen sind therapeutisch tätige Psychologen mit astrologischer Spezialisierung (Hover 2001, 1). Soweit diese sich als Diplom-Psychologen bezeichnen, ist ein Universitätsexamen mit Studiengängen von mindestens 4 Jahren vorausgesetzt ${ }^{11}$, so dass die oberste Stufe reglementierter Berufe i.S. des Kommissionsvorschlags 2002 in Betracht kommt. Dürfen zu dieser Berufsgruppe Migranten aus anderen EU-Ländern mit 3jährigem Diplomstudiengang und ohne die für Freiberufler typische Mitgliedschaft in einem Berufsverband hinzutreten, so droht die im DAV über viele Jahre aufgebaute Abgrenzung gegenüber nicht wissenschaftlich und nicht fachlich eigenverantwortlichen tätigen PsychoAstrologen durchbrochen zu werden. Deshalb wurde dem EU-Parlament nahe gelegt, die Anerkennung nicht freiberuflicher Qualifikationen nicht vorzusehen.

Das Parlament hat daraufhin in seiner Entschließung v. Febr. 2004 vorgeschlagen, den Begriff des „reglementierten Berufs“ in Art. 3 Abs. 1 lit. a durch die in Deutschland geläufigen Freiberufskriterien zu ersetzen. V.a. aber wird Art. 13 Abs.1 lit.b Kommissionsvorschlag 2002 dahingehend abgeändert, dass keine stufenweise Unterschreitung der Ausbildungsniveaus zugelassen, sondern deren Entsprechung verlangt wird. Das entspricht jedenfalls im Ansatz den Wünschen des DAV, denn durch die Niveauentsprechung würde sichergestellt, dass der Migrant den Ausbildungsanforderungen entspricht, die in Deutschland für die Anerkennung als Freiberufler erfordert wird.

\subsection{Kommissionsentwurf 2004 und Kritik}

Der Kommissionsvorschlag 2004 ist dem nicht gefolgt und hat an der 1-stufigen Unterschreitungsmöglichkeit festgehalten. Die Neufassung des Art. 13 des Parlamentsvorschlags ist im Kommissionsvorschlag 2004 nicht nur nicht übernommen, sondern auch mit keinerlei Kompromissregelung aufgenommen worden, obgleich die angesprochenen Risiken eines Parlamentsdissenses im Mitentscheidungsverfahren bestehen. Offenbar will man im gegenwärtigen Stand des Verfahrens den eigenen Standpunkt durchhalten, um die Grundkonzeption nicht zu verfälschen.

Zur Begründung gibt die Kommission an, dass es sich nicht um eine freiberufsspezifische Regelung handelt. Die Grundkonzeption der Kommission leugnet eine Sonderstellung Freier Berufe. $^{12}$ Dahinter steht wohl auch die Einschätzung, dass es in Europa keine einheitliche Begriffs- oder Typusbildung Freier Berufe gibt, und man sich scheut, die deutsche Umschreibung gemäß $\S 1$ PartGG und § 18 EStG den Rechten der übrigen EU-Mitgliedstaaten vorzugeben (Mann 2004, 616). Doch kann von einer deutschrechtlichen Überfremdung v.a. deshalb gar keine Rede sein, weil das hierzulande geltende Freiberufskonzept gemeinsame Wurzeln mit der sozialgeschichtlich gut erforschten Professionalisierung wissenschaftlich fundierter Berufe in den USA, England, Frankreich und vielen anderen Ländern hat (vgl. - grundlegend - Konze/ Kocka 1992).

Für den vorliegenden Beitrag konnte bisher nicht in Erfahrung gebracht werden können, ob auch Stellungnahmen aus anderen Ländern das EU-Parlament zu einer derart grundlegenden Kritik am Ansatz der Kommission veranlasst haben. Die Stellungnahmen werden von den EU-Stellen nicht veröffentlicht, so dass man insoweit auf Anfragen bei den Schwesterorgani-

\footnotetext{
11 Vgl. Art. 86 Abs. 1 Bay.HochschulG i.d.F. v. 2.10.1998, GVBl. 740 ff.; andere Bundesländer haben übereinstimmende Regelungen, s. Herrmann 2003a, 64.

12 Kommission, Geänderter Richtlinienvorschlag, KOM (2004) 317 endg., 46.
} 
sationen des BfB in den EU-Mitgliedstaaten angewiesen ist, die am ehesten dafür in Betracht kommen. Dafür wären Anfragen mit Fragebögen bei den Dachverbänden Freier Berufe in allen alten EU-Mitgliedstaaten geeignet, da die Stellungnahmen vor dem Beitritt der neuen Staaten abzugeben waren. Hypothetisch kann davon ausgegangen werden, dass v.a. die Stellungnahmen Spaniens und Italiens Fakten und Meinungen enthalten, die denen des BfB vergleichbar sind. Denn diese beiden Länder haben Kammersysteme, die denen der BRD weit mehr ähneln als das Organisationsrecht Englands, Irlands und der Niederlande. Aber auch Österreich kommt natürlich wegen der Nähe zum deutschen Wirtschafts- und Freiberufsrecht in Betracht.

\subsection{Zentrale Regelung zur Eignungsprüfung}

Um zu krassen Gegensätzen entgegenzuwirken, wird bei Abweichung der Ausbildungsdauer von 1 Jahr die Möglichkeit von Anpassungslehrgängen oder Eignungsprüfungen eröffnet, wobei die Entscheidung über eine der beiden Alternativen von der Kommission in die Hände des Migranten selbst gelegt werden soll ${ }^{13}$, nach Ansicht des Parlaments der Regulierung des Gastlandes vorbehalten bleibt. ${ }^{14}$ Es scheint, dass hier angesichts der aufgezeigten Möglichkeiten des Parlaments im Mitentscheidungsverfahren Durchsetzungsmöglichkeiten für den Standpunkt des Parlaments bestehen.

Zweifellos können auch Anpassungslehrgänge sichern, dass die für freiberufliche Tätigkeiten typische Fachkompetenz gewahrt wird, sofern sie mit entsprechenden Inhalten versehen und mit Abschlussprüfungen verbunden sind. Aber die Kontrolle darüber sollte dringend übergeordneten Stellen übertragen, und nicht der opportunistischen Entscheidung des Betroffenen überlassen werden. Auch insoweit kann vielleicht zur Stärkung des Standpunktes des EUParlaments beigetragen werden.

Gegen die Lösung der Kommission spricht entscheidend, dass das Konzept europaweiter Vereinheitlichung der Migrationsbedingungen beschädigt würde. Dagegen lässt sich auch nicht einwenden, der so genannte Systemwettbewerb ${ }^{15}$ könne dazu führen, dass sich längerfristig die am besten geeignete Regulierung durchsetzen wird, denn bei dieser Norm geht es um eine wettbewerbliche Rahmenbedingung, deren Setzung nicht den Marktteilnehmern selbst überlassen bleiben darf. Obgleich also nur die zentrale Regelung durch den europäischen Richtliniengeber bleibt, ist es nicht ausgeschlossen, den am Systemwettbewerb Beteiligten eine Auswahl einzuräumen, wenn dieser ein klarer Rahmen vorgegeben wird. Dieser könnte, wie folgt, aussehen:

In vielen Fällen wird der Niveau-Unterschied, der durch die Anerkennungsregeln des Richtlinienvorschlags überwunden werden kann, im Zielland gerade dadurch bewirkt, dass ein Ausbildungsabschluss verlangt wird, der in einer qualifizierenden Abschlussprüfung besteht. Ist dies der Fall, so muss vom Migranten entweder ein vergleichbarer Prüfungsabschluss aus dem Herkunftsland verlangt werden, oder die Eignungsprüfung ist einheitlich vorzuschreiben.

Mit einer solchen Regelung kann dem Bedenken der Kommission in gewissem Umfang Rechnung getragen werden, ein Wahlrecht der Zielländer könne zu neuen nationalen Unter-

\footnotetext{
13 Art. 14 Abs. 2 Kommissionsvorschlag 2002; Begr. des geänderten Richtlinienentwurfs, KOM (2004) 317 endg., $48 \mathrm{f}$.

14 Abänderungsvorschlag Nr. 65.

15 Dazu näher sogleich zu 4.2.
} 
schieden und damit zu ungleichen Marktzutrittsbedingungen im Binnenmarkt führen. Denn ein Wahlrecht entfiele. Die Anknüpfung an vorhandene Prüfungsanforderungen, die sich im Zielland unter den herkömmlichen Wettbewerbsbedingungen bewährt hat, spricht gegen binnenmarktbeschränkende Wirkungen. Die hier vorgeschlagene Regelung kann u.U. noch dahin ergänzt werden, dass ein Vorbehalt zur Wirksamkeit funktionsfähigen nationalen und internationalen Wettbewerbs auf den betreffenden Märkten des Ziellandes vorsieht. Denn soweit dies der Fall ist, scheint den von der Kommission befürchteten Marktabschottungsgefahren hinreichend entgegengewirkt.

\section{$4 \quad$ Clementi-Reformen im Systemwettbewerb}

\subsection{Clementi-Bericht und Regierungsplan 2005}

In diesem Teil der Untersuchung sollen die rechtsvergleichenden Entwicklungen betrachtet werden. Im Juli 2003 erklärte das englische Ministerium für Verfassungsangelegenheiten offiziell seine fundamentale Unzufriedenheit mit der Arbeit der Berufsorganisationen und bezeichnete deren System als „überholt, unflexibel, überkomplex, nicht ausreichend verantwortlich und nicht ausreichend transparent“ (Zit. n. Hellwig 2005, 1218). Daraufhin wurde Sir David Clementi mit einem Reformgutachten beauftragt, das dieser im Dezember 2004 mit für die Law Society und den Bar Council of England and Wales größtenteils niederschmetternden Analysen und Vorschlägen vorgelegt hat. Im März 2005 hat die englische Regierung sich dieser Sicht im Kern angeschlossen und entsprechende Reformen angekündigt. Diese werden im Folgenden abkürzend Clementi-Reformen genannt.

Im Kern geht es bei den Clementi-Reformen um folgendes:

Beide kammerähnlichen Rechtsanwaltsorganisationen sollen die bisherigen Regulierungsbefugnisse der Selbstverwaltung verlieren. Statt dessen wird ein Legal Services Board als staatliches Zentralorgan eingerichtet, das die für notwendig erachteten Regulierungen entweder selbst erlässt, oder an die bestehenden Berufsorganisationen delegieren kann, wenn es sich davon überzeugt hat, dass diese über hinreichende Regulierungskompetenz verfügen. Derzeit wird dies offenbar nicht angenommen. Hellwig hat diese Entwicklung treffend dahingehend kommentiert, dass „die Selbstregulierung, weil sie versagt hat, erst aufgehoben und dann unter Kuratel gestellt“" werden soll (Hellwig 2005, 1223). Darüber hinausgehend kann man auf die Einrichtung des zentralen Staatsorgans und dessen primäre Regulierungszuständigkeit abstellen und darin echte Aufgaben der Wirtschaftslenkung erkennen. Mit Blick auf die mögliche Rückübertragung der Regulierungsbefugnisse auf die bestehenden Berufsvertretungen, wenn deren hinreichende Regulierungskompetenz vom Board festgestellt ist, scheint eine Art gelenkter Selbstverwaltung vorzuliegen, die in der Geschichte der Freiberufsrecht ebenso wie in der der Selbstverwaltungsrechte, soweit ersichtlich, bisher völlig unbekannt ist.

Die Entwicklung hat trotz ihrer Neuartigkeit bereits ausgestrahlt auf die Verbandsrechte in Irland, den Niederlanden und Frankreich ${ }^{16}$, und ebenso auch auf andere Freiberufsrechte. Beispielsweise befasst sich die deutsche Monopolkommission seit Dezember 2004 mit dem Thema „Regulierung im Bereich der Freien Berufe“ und hat hierzu einen umfangreichen Fragenkatalog an Kammern und Verbände Freier Berufe versendet, der u.a. auch die Aufgaben

16 Näheres bei Hellwig, ebd. 
und rechtliche Organisation der Berufsvertretungen betrifft (vgl. Hellwig, a.a.O., 1218). Das kann hier im Einzelnen natürlich nicht dargestellt werden. Doch zeigen sich deutlich Kräfte des so genannten Systemwettbewerbs, von denen im Folgenden noch näher zu sprechen ist.

Vorerst ist festzuhalten, dass das englische Freiberufsrecht von der überkommenen Selbstverwaltung mit Ansätzen zur Öffnung für Wettbewerbseinflüsse abgehen wird und stattdessen entweder auf staatliche Regulierung umstellt, oder eine staatlich gelenkte Verbandsregulierung zulässt. Dadurch werden zwar die überkommenen Professionalisierungsmerkmale der Unabhängigkeit, Eigenverantwortlichkeit, persönlicher Aufgabenwahrnehmung und wissenschaftlichen Fundierung nicht vollständig aufgegeben. Aber die staatlichen Aufsichtsbefugnisse sollen derart ausgeweitet werden, dass von einem Prinzip der Selbstverwaltung kaum noch die Rede sein kann.

\subsection{Gegenkräfte im deutschen System der Selbstverwaltung}

In Deutschland ist diese Entwicklung bisher nur auf Ablehnung gestoßen (vgl. nur Hellwig, a.a.O., 1218 ff). Obgleich auch Vorzüge englischer Rechtsvergleichung, ist ein derartiges Radikalkonzept kritisch zu beäugen. Doch geht es nicht um persönliche Meinungen, sondern darum, ob das Konzept gelenkter Selbstverwaltung im so genannten Regulierungswettbewerb erfolgreich sein wird. Dabei handelt es sich zwar um eine noch recht junge Entdeckung der Wettbewerbswissenschaft. Sie hat aber im Unternehmensrecht bereits derart viel Bestätigung gefunden, dass auch ihre Validität in so unterschiedlichen Bereichen wie denen des Arbeitsrechts, Umweltschutzes und der Regulierung des Bank- und Kapitalmarktrechts angenommen wird. ${ }^{17}$ Es liegt deshalb nahe, entsprechende wettbewerbliche Regulierungsfunktionen auch im allgemeinen Verbandsrecht und im Recht der Freiberufsverbände heute anzunehmen. ${ }^{18}$

Folgende Grundannahmen sind wesentlich und für das Recht Freier Berufe relevant:

- Wettbewerb i.S. der unsichtbaren Hand von Adam Smith funktioniert nicht nur im Überlebenskampf gewinnorientierter Unternehmen, sondern auch im Wettstreit staatlicher oder kommunaler Regulierer um Unternehmensansiedlungen, die von Standortvorteilen für international mobile Marktteilnehmer abhängig gemacht werden;

- $\quad$ Dazu gehört nicht nur der bekannte Delaware-Effekt des Aktienrechts, der auf extrem niedrige Regulierungsdichte ausgerichtet ist (sog. race to the buttom), sondern auch der Trend in die entgegen gesetzte Richtung (sog. California-Effekt) ${ }^{19}$;

- Bei hoher internationaler Mobilität kommt deshalb ein funktionsfähiger Wettbewerb mit attraktiven regulatorischen Systemen zustande, bei dem sich schließlich das beste Regelungsset durchsetzt (R. van den Berg 2000, 435).

Ohne weitere theoretische Vertiefung kann auf empirische Erkenntnisse des Systemwettbewerbs der corporate governance verwiesen werden, die das wettbewerbliche Ringen zwischen U.S.-amerikanischen und europäischen Aktienrechtsregimen belegen (P. Witt 2000, 159, 163). Hauptsächlich geht es hier um die Optimierung von Systemen mit Shareholder-Value-

17 Vgl. nur die Fallstudien bei D.D. Murphy 2004, 45 ff..

18 Vgl. nur Ogus 1995, R. van den Berg 2000, 435; ders. 2004, 10, passim; Herrmann 2004, 10.

19 Dazu und zum weiteren sog. Japanese effect s. nochmals Mürphy 2004, 45 ff. 
Dominanz oder mit Beteiligung von Gläubiger- und Arbeitnehmerinteressen (stakeholders). Im hier interessierenden Bereich der professional governance stehen sich hauptsächlich Regime der beruflichen Selbstverwaltung und solche mit dominantem Staatseinfluss gegenüber. Der Systemwettbewerb lässt die englisch-irische Seite derzeit zu stärker dirigistischen Formen greifen, während sich die dezentralen Modelle offenbar in Deutschland hartnäckiger halten, obgleich hierzulande traditionell ein viel höherer Anteil hoheitlicher Aufsichts- und Mitwirkungsbefugnisse gegeben ist.

Besonders eindringlich hat unlängst der Präsident des Rates der Anwaltschaften der EU, Hellwig, dazu aufgerufen, dem englischen Regulierungstrend der Clementi-Reformen in Deutschland entgegenzutreten. Dies könne aber nur geschehen, wenn die freiberufliche Selbstverwaltung unter Beweis stelle, dass sie sozusagen von innen heraus in der Lage sei, die erkannten Reformbedarfe zu leisten. Konkret müsse etwa das Verbot der Werbung um Praxis gelockert und auf Regeln gegen die Ausnutzung von Schock- oder Notsituationen begrenzt werden (,ambulance chasing“). ${ }^{20}$ Auch sei die Abschaffung gesetzlich fixierter Beratungshonorare durch das neue Rechtsanwaltsvergütungsgesetz (RVG) ein Schritt in die richtige Richtung. Doch sei der Vorschlag der Monopolkommission zu bedenken, die verbleibende Festpreisregelung für Prozessvertretungen dahingehend abzuändern, dass nur noch Obergrenzen für von der Gegenpartei zu erstattende Gebühren gelten (Hellwig 2005, 1218). Lockerungen zu den Sozietätsverboten werden ebenso empfohlen (ebd., 1218) wie erhebliche Erleichterungen zur Aufnahme von Eigenkapital bei nicht berufsangehörigen Investoren (ebd., 1221), die Bereinigung der Regulierungsaufgaben der Kammern und Verbände von wirtschaftlichen Interessenkonflikten, Verbesserungen der „demokratischen Repräsentativität und Transparenz“ (ebd., 1224 f.). Man erkennt unschwer die Ähnlichkeit zu Anforderungen der corporate governance und der professional governance.

Ob man deshalb aber mit Clementi so weit gehen sollte, dass die Konzeption des Rechtsanwalts als Organ der Rechtspflege hinter der der „Legal Services Industry“ zurückzutreten habe, hält Hellwig für „problematisch“ (ebd., 1222). Und v.a. sei die Clementi-Bewegung in Europa ernst zu nehmen und innerhalb der Berufsorganisationen ,...zu überlegen, was zu tun ist, damit es nicht zu ähnlichen Entwicklungen bei uns kommt“ (ebd., 1223), obgleich bereits 2003 eine Reihe von SPD-Bundestagsabgeordneten sich für die Abschaffung der verkammerten Selbstverwaltung öffentlich ausgesprochen hat, und die FDP sich im gerade zu Ende gegangenen NRW-Wahlkampf diesem Ziel verschrieben hat.

Die Arbeiten des Autors sind seit Jahren auf die von Hellwig formulierten Ziele und damit auf Bewahrung des Systems der Selbstverwaltung ausgerichtet. Auch ist derzeit eine gewisse Gefahr zu sehen, dass der internationale Systemwettbewerb die englische Staatskuratel über den Berufsorganisationen bevorzugt. Doch sind im Unterschied zur Mehrzahl der Bewahrer deutscher Tradition zu sehen, dass kräftige Innovationsimpulse aus England zu uns herüberkommen. Wir sollten so viel wie möglich von ihnen lernen, ohne die Vorteile der eigenen Entwicklung gänzlich aufzugeben.

5 Fazit

Abschließend kann zusammengefasst werden:

20 Hellwig, NJW 2005, 1217, 1219; dazu siehe oben zu 2. 
1. Werbeverbote von Freiberufskammern verletzen häufig das EU-Kartellrecht und laufen deshalb Gefahr, durch die neue Kartellrechtspolitik seit Monti angegriffen zu werden. Die Kommission ist aber gut beraten, wenn sie nicht mit Abstellensverfügungen reagiert, sondern Verpflichtungszusagen entgegennimmt, die kartellrechtskonforme Werbeverbote beinhalten (Beispiel: ambulance chasing).

2. Die EU-Richtlinie zur Anerkennung von Berufsqualifikationen sollte die Freiberuflichkeit als Typus reglementierter Berufe vorsehen und bei Niveau-Unterschreitungen von Immigranten eine Wahlmöglichkeit zwischen Anpassungslehrgängen oder Eignungsprüfungen vorsehen, die objektiv danach konditioniert ist, dass der Niveau-Unterschied im Zielland nur bei Ablegung einer oder mehrerer Abschlussprüfungen überwunden werden kann. Die Entscheidung sollte nicht dem Migranten überlassen bleiben, sondern dem Mitgliedstaat obliegen.

3. Derzeit besteht eine erhebliche Gefahr, dass Deutschland im internationalen Systemwettbewerb das englische Modell einer Staatskuratel über den Berufsorganisationen á la Clementi bevorzugt. Doch gehen von den Clementi-Reformen nicht nur Bedrohungen des deutschen Selbstverwaltungsmodells aus, sondern es werden auch kräftige Innovationsimpulse ausgelöst, von denen wir so viel wie möglich von ihnen lernen sollten, ohne die Vorteile der eigenen Entwicklung zu weitgehend aufzugeben.

4. Insgesamt scheint sich der schon in den 90er Jahren beobachtete Trend von „harten“ zu „weichen“ Kammerformen fortzusetzen und mit Elementen der Professional Governance angereichert zu werden. Ob darin eine überlegene Alternative zu englischen Modell gelenkter Selbstverwaltung liegt, kann nicht prognostiziert werden, sondern muss sich nur im Systemwettbewerb staatlicher und halb-staatlicher Regulierer erweisen.

\section{Literatur}

Bundesverband Freier Berufe (2002), Resolution der Mitgliederversammlung des Bundesverbandes der Freien Berufe v. 19.6.2002, Internetversion.

Dreher, M. (2004), Kartellrechtscompliance in der Versicherungswirtschaft, in: Versicherungsrecht 2004, $1 \mathrm{ff}$.

Hellwig, H.-J. (2005), Perspektiven der deutschen Anwaltschaft ex Europa, in: Neue Juristische Wochenschrift - NRW, 2005, 1217.

Herrmann, H. (2004), Antitrust Law Compliance and Professional Governance, in: Ehlermann (Hrsg.), European Competition Law Annual 2004, 2006, 101 ff.

Herrmann, H. (2003), Europäische Entwicklungstrends des Freiberufsrechts mit Blick auf die Astrologie im DAV, Kurzgutachten, erstellt im Auftrag des Deutschen Astrologenverbandes.

Herrmann, H. (2003a), Freiberufsrecht psychologischer Gutachter und Berater, maschinenschriftliche Fassung.

Herrmann, H. (1996), Recht der Kammern und Verbände Freier Berufe - Europäischer Ländervergleich und USA, Schriften des Forschungsinstituts Freie Berufe der Universität Lüneburg Nr. 8, NOMOS-Verlagsgesellschaft, Baden Baden.

Hover, D. (2001), Astrologie und Beratung - Ergebnisse einer Befragung unter Astrologen, in: Meridian, 5/2001, 44-48.

Kleine-Cosack, M. (2004), Das Werberecht der rechts- und steuerberatenden Berufe, 2. Auflage, Beck Juristischer Verlag, München. 
Conze, W. und J. Kocka (Hrsg.) (1992), Bildungsbürgertum, Teil I, 2. Auflage, Klett-Cotta, Stuttgart.

Mann, T. (2004), Randnotizen zum Richtlinienentwurf über die Anerkennung von Berufsqualifikationen, in: EuZW, 2004, 615-619.

Murphy, D. D. (2004), The Structure of Regulatory Competition, Oxford University Press, Oxford.

Ogus, A. (1995), Rethinking Self-Regulation, in: Oxford Journal of Legal Studies, Vol. 15, 97-108.

Parker, C. (2001), The Open Corporation - Effective Self-Regulation \& Democracy, Cambridge University Press, Cambridge.

Ringleb, H.-M., Kremer, T., Lutter, M. und A. von Werder (2003), Deutscher Corporate Governance Kodex, Kommentar, München

Van den Bergh R. (2004), Towards Efficient Self-Regulation in Markets for Professional Services, in: Ehlermann (Hrsg.), European Competition Law Annual 2004, 2006, 155 ff.

Van den Bergh, R. (2000), Towards an institutional legal framework for regulatory competition, 435. 


\section{Forschungsinstitut Freie Berufe (FFB), Universität Lüneburg Publikationen}

\section{FFB-Jahresberichte}

FFB-Jahresbericht 2005/06, FFB-Jahresbericht 2003/04, FFB-Jahresbericht 2001/02, FFB-Jahresbericht 1999/00, FFB-Jahresbericht 1997/98, FFB-Jahresbericht 1996, FFB-Jahresbericht 1995, FFBJahresbericht 1994, FFB-Jahresbericht 1993, FFB-Jahresbericht 1992, FFB-Jahresbericht 1991, FFB: 10 Jahre Forschung und Lehre 1989-1999, FFB-Forschung und Lehre 1989-1998, FFB-Forschung und Lehre 1989-1997, FFB-Forschung und Lehre 1989-1996.

\section{$2 \quad$ FFB-Bücher in der FFB-Schriftenreihe}

Merz, J. (Hrg.), 2008, Freie Berufe - Einkommen und Steuern (FB€St), FFB-Schriften Nr. 16, 301 Seiten, ISBN 978-3-8329-3210-7, NOMOS Verlagsgesellschaft, Baden-Baden.

Preis: € 54,-

Ackermann, D., 2006, Erfolgsfaktoren für Existenzgründung und Existenzsicherung freiberuflich tätiger Ärzte, FFB-Schriften Nr. 15, 399 Seiten, ISBN 3-8329-2060-9, NOMOS Verlagsgesellschaft, Baden-Baden.

Preis: € 69,-

Ehling, M. und J. Merz, 2003, Neue Technologien in der Umfrageforschung, FFB-Schriften Nr. 14, 181 Seiten, ISBN 3-7890-8241-4, NOMOS Verlagsgesellschaft, Baden-Baden.

Preis: € 31,-

Merz, J., 2003, Freie Berufe im Wandel der Märkte, FFB-Schriften Nr. 13, 168 Seiten, ISBN 3-7890-8107-8, NOMOS Verlagsgesellschaft, Baden-Baden.

Preis: € 29,-

Merz, J., 2001, Existenzgründung 2 - Erfolgsfaktoren und Rahmenbedingungen, FFB-Schriften Nr. 12, 232 Seiten, ISBN 3-7890-7462-4, NOMOS Verlagsgesellschaft, Baden-Baden.

Preis: € 40,- / € 71,- (i. Vb. mit Band 1)

Merz, J., 2001, Existenzgründung 1 - Tips, Training und Erfahrung, FFB-Schriften Nr. 11, 246 Seiten, ISBN 37890-7461-6, NOMOS Verlagsgesellschaft, Baden-Baden. $\quad$ Preis: € 40,- / € 71,- (i. Vb. mit Band 2)

Merz, J. und M. Ehling, 1999, Time Use - Research, Data and Policy, FFB-Schriften Nr. 10, 571 Seiten, ISBN 3-7890-6244-8, NOMOS Verlagsgesellschaft, Baden-Baden.

Preis: € 49 ,-

Herrmann, H. und J. Backhaus, 1998, Staatlich gebundene Freiberufe im Wandel, FFB-Schriften Nr. 9, 234 Seiten, ISBN 3-7890-5319-8, NOMOS Verlagsgesellschaft, Baden-Baden.

Preis: € 34,-

Herrmann, H., 1996, Recht der Kammern und Verbände Freier Berufe, Europäischer Ländervergleich und USA, FFB-Schriften Nr. 8, 596 Seiten, ISBN 3-7890-4545-4, NOMOS Verlagsgesellschaft, Baden-Baden.

Preis: € 56,-

Merz, J., Rauberger, T. K. und A. Rönnau, 1994, Freie Berufe in Rheinland-Pfalz und in der Bundesrepublik Deutschland - Struktur, Entwicklung und wirtschaftliche Bedeutung, FFB-Schriften Nr. 7, 948 Seiten, ISBN 3-927816-27-2, Lüneburg.

Preis: € 95,-

Forschungsinstitut Freie Berufe (FFB) (Hrsg.), erstellt vom Zentrum zur Dokumentation für Naturheilverfahren e.V. (ZDN), 1992, 1993, Dokumentation der besonderen Therapierichtungen und natürlichen Heilweisen in Europa, Bd. I, 1. Halbband, 842 Seiten, Bd. I, 2. Halbband, 399 Seiten, Bd. II, 590 Seiten, Bd. III, 272 Seiten, Bd. IV, 419 Seiten, Bd. V, 1. Halbband, 706 Seiten, Bd. V, 2. Halbband, 620 Seiten, ISBN 388699-025-7, Lüneburg (nur zu beziehen über das Zentrum zur Dokumentation für Naturheilverfahren e.V. ZDN, Hufelandstraße 56, 45147 Essen, Tel.: 0201-74551).

Preis: € 385,-

Sahner, H. und A. Rönnau, 1991, Freie Heilberufe und Gesundheitsberufe in Deutschland, FFB-Schriften Nr. 6, 653 Seiten, ISBN 3-927816-11-6, Lüneburg.

Preis: € 58,- 
Burmester, B., 1991, Ausbildungsvergleich von Sprachtherapeuten, FFB-Schriften Nr. 5, 54 Seiten, ISBN

3-927816-10-8, Lüneburg.

Preis: € 9,-

Sahner, H., 1991, Freie Berufe in der DDR und in den neuen Bundesländern, FFB-Schriften Nr. 4, 177 Seiten, ISBN 3-927816-09-4, Lüneburg.

Preis: € 25,-

Trautwein, H.-M., Donner, H., Semler, V. und J. Richter, 1991, Zur tariflichen Berücksichtigung der Ausbildung, der Bereitstellung von Spitzenlastreserven und der Absicherung von Beschäftigungsrisiken bei Seelotsen, mit dem Anhang Steuerliche Aspekte des tariflichen Normaleinkommens der Seelotsen, FFBSchriften Nr. 3, 183 Seiten, ISBN 3-927816-07-8, Lüneburg.

Preis: € 19,-

Sahner, H. und F. Thiemann, 1990, Zukunft der Naturheilmittel in Europa in Gefahr? FFB-Schriften Nr. 2, 81 Seiten, ISBN 3-927816-06-X, Lüneburg.

Preis: € 6,-

Sahner, H., Herrmann, H., Rönnau, A. und H.-M. Trautwein, 1989, Zur Lage der Freien Berufe 1989, Teil III, FFB-Schriften Nr. 1, 167 Seiten, ISBN 3-927816-04-3, Lüneburg.

Preis: $€ 25$,-

Sahner, H., Herrmann, H., Rönnau, A. und H.-M. Trautwein, 1989, Zur Lage der Freien Berufe 1989, Teil II, FFB-Schriften Nr. 1, 955 Seiten, ISBN 3-927816-02-7, Lüneburg.

Preis: € 20,- / € 35,- (i. Vb. mit Teil I)

Sahner, H., Herrmann, H., Rönnau, A. und H.-M. Trautwein, 1989, Zur Lage der Freien Berufe 1989, Teil I, FFB-Schriften Nr. 1, 426 Seiten, ISBN 3-927816-01-9, Lüneburg.

Preis: € 20,- / € 35,- (i. Vb. mit Teil II)

\section{$3 \quad$ FFB-Bücher}

Merz, J. (Hrg.), 2007, Fortschritte der MittelstandsForschung, Merz, J., Schulte, R. and J. Wagner (Series Eds.), Entrepreneurship, Professions, Small Business Economics, CREPS-Schriftenreihe Vol. 3, 520 Seiten, Lit Verlag, ISBN 3-8258-0265-3, Münster.

Preis: $€ 34,90$

Zwick, M., 2007, Alternative Modelle zur Ausgestaltung von Gemeindesteuern, Statistisches Bundesamt - Statistik und Wissenschaft - Band 8, 307 Seiten, ISBN 978-3-8246-0791-4,

Preis: $€ 24,80$

Zwick, M., J. Merz u.a., 2007, MITAX - Mikroanalysen und Steuerpolitik, Statistisches Bundesamt - Statistik und Wissenschaft - Band 7, 261 Seiten, ISBN 978-3-8246-0790-7,

Preis: € 24,80

Schulte, R. (Hrg.), 2005, Ergebnisse der MittelstandsForschung, Merz, J., Schulte, R. and J. Wagner (Series Eds.), Entrepreneurship, Professions, Small Business Economics, CREPS-Schriftenreihe Vol. 2, 432 Seiten, Lit Verlag, ISBN 3-8258-8916-5, Münster.

Preis: $€ 34,90$

Merz, J., D. Hirschel und M. Zwick, 2005, Struktur und Verteilung hoher Einkommen - Mikroanalysen auf der Basis der Einkommensteuerstatistik, Lebenslagen in Deutschland, Der zweite Armuts- und Reichtumsbericht der Bundesregierung, Bundesministerium für Gesundheit und Soziale Sicherung, Berlin (http://www.bmgs.bund.de/download/broschueren/A341.pdf)

Merz, J. und J. Wagner (Hrg.), 2004, Perspektiven der MittelstandsForschung - Ökonomische Analysen zu Selbständigkeit, Freien Berufen und KMU, Merz, J., Schulte, R. and J. Wagner (Series Eds.), Entrepreneurship, Professions, Small Business Economics, CREPS-Schriftenreihe Vol. 1, 520 Seiten, Lit Verlag, ISBN 3-8258-8179-2, Münster.

Preis: $€$ 39,90

Merz, J. und M. Zwick (Hrg.), 2004, MIKAS - Mikroanalysen und amtliche Statistik, Statistisches Bundesamt (Serie Editor), Statistik und Wissenschaft, Vol. 1, 318 Seiten, ISBN 3-8246-0725-5, Wiesbaden.

Preis: € 24,80

Hirschel, D., 2004, Einkommensreichtum und seine Ursachen - Die Bestimmungsfaktoren hoher Arbeitseinkommen, Hochschulschriften Band 82, 416 Seiten, Metropolis-Verlag Marburg, ISBN 3-89518-441-1.

Merz, J., 2001, Hohe Einkommen, ihre Struktur und Verteilung, Lebenslagen in Deutschland, Der erste Armutsund Reichtumsbericht der Bundesregierung, Bundesministerium für Arbeit und Sozialordnung, 128 Seiten, Bonn, zu beziehen über: Bundesanzeiger Verlagsgesellschaft mbH, Südstraße 119, 53175 Bonn. 
Ehling, M. und J. Merz u. a., 2001, Zeitbudget in Deutschland - Erfahrungsberichte der Wissenschaft, Band 17 der Schriftenreihe Spektrum Bundesstatistik, 248 Seiten, Metzler-Poeschel Verlag, ISBN 3-8246-0645-3, Stuttgart.

Preis: € 16,-

Krickhahn, T., 1995, Die Verbände des wirtschaftlichen Mittelstands in Deutschland, 351 Seiten, DUV Deutscher Universitäts Verlag, ISBN 3-8244-0245-9, Wiesbaden.

Preis: € 62,-

Spahn, P. B., Galler, H. P., Kaiser, H., Kassella, T. und J. Merz, 1992, Mikrosimulation in der Steuerpolitik, 279 Seiten, Springer Verlag, ISBN 3-7908-0611-0, Berlin.

Preis: € 45,-

\section{$4 \quad$ FFB-Reprints}

Merz, J., Kumulation von Mikrodaten - Konzeptionelle Grundlagen und ein Vorschlag zur Realisierung,; in: Allgemeines Statistisches Archiv, Issue 88, S. 451-472, 2004

FFB-Reprint Nr. 35

Merz, J., Einkommens-Reichtum in Deutschland - Mikroanalytische Ergebnisse der Einkommensteuerstatistik für Selbständige und abhängig Beschäftigte, in: Perspektiven der Wirtschaftspolitik, Vol. 5, Issue 2, S. 105-126, 2004

FFB-Reprint Nr. 34

Merz, J., Vorgrimler, D. und M. Zwick, Faktisch anonymisiertes Mikrodatenfile der Lohn- und Einkommensteuerstatistik 1998, in: Wirtschaft und Statistik, Issue 10, S. 1079-1091, 2004

FFB-Reprint Nr. 33

Ackermann, D., Merz, J. and H. Stolze, Erfolg und Erfolgsfaktoren freiberuflich tätiger Ärzte - Ergebnisse der FFB-Ärzteumfrage für Niedersachsen, in: Merz, J. and J. Wagner (Eds.), Perspektiven der MittelstandsForschung - Ökonomische Analysen zu Selbständigkeit, Freien Berufen und KMU, Merz, J., Schulte, R. and J. Wagner (Series Eds.), Entrepreneuship, Professions, Small Business Economics, CREPSSchriftenreihe Vol. 1, Lit Verlag, Münster, pp. 165-190, 2004

FFB-Reprint Nr. 32

Hirschel, D. und J. Merz, Was erklärt hohe Arbeitseinkommen Selbständiger - Eine Mikroanalyse mit Daten des Sozioökonomischen Panels, in: Merz, J. and J. Wagner (Eds.), Perspektiven der MittelstandsForschung Ökonomische Analysen zu Selbständigkeit, Freien Berufen und KMU, Merz, J., Schulte, R. and J. Wagner (Series Eds.), Entrepreneuship, Professions, Small Business Economics, CREPS-Schriftenreihe Vol. 1, Lit Verlag, Münster, pp. 265-285, 2004

FFB-Reprint Nr. 31

Burgert, D. und J. Merz, Wer arbeitet wann? - Arbeitszeitarrangements von Selbständigen und abhängig Beschäftigten: Eine mikroökonometrische Analyse deutscher Zeitbudgetdaten, in: Merz, J. and J. Wagner (Eds.), Perspektiven der MittelstandsForschung - Ökonomische Analysen zu Selbständigkeit, Freien Berufen und KMU, Merz, J., Schulte, R. and J. Wagner (Series Eds.), Entrepreneuship, Professions, Small Business Economics, CREPS-Schriftenreihe Vol. 1, Lit Verlag, Münster, pp. 303-330, 2004

FFB-Reprint Nr. 30

Merz, J. und M. Zwick, Hohe Einkommen - Eine Verteilungsanalyse für Freie Berufe, Unternehmer und abhängige Beschäftigte, in: Merz, J. and M. Zwick (Eds.), MIKAS - Mikroanalysen und amtliche Statistik, Statistik und Wissenschaft, Vol. 1, pp. 167-193, 2004

FFB-Reprint Nr. 29

Merz, J., Schatz, C. and K. Kortmann, Mikrosimulation mit Verwaltungs- und Befragungsdaten am Beispiel ,Altersvorsorge in Deutschland 1996' (AVID ,96), in: Merz, J. und M. Zwick (Hg.), MIKAS - Mikroanalysen und amtliche Statistik, Statistik und Wissenschaft, Vol. 1, S. 231-248, 2004 FFB-Reprint Nr. 28

Merz, J., Was fehlt in der EVS ? - Eine Verteilungsanalyse hoher Einkommen mit der verknüpften Einkommensteuerstatistik für Selbständige und abhängig Beschäftigte, in: Jahrbücher für Nationalökonomie und Statistik, Vol. 223/1, p. 58-90, 2003

FFB-Reprint Nr. 27

Merz, J. und M. Zwick, Verteilungswirkungen der Steuerreform 2000/2005 im Vergleich zum ,Karlsruher Entwurf' - Auswirkungen auf die Einkommensverteilung bei Selbständigen (Freie Berufe, Unternehmer) und abhängig Beschäftigte, in: Wirtschaft und Statistik, 8/2002, p. 729-740, 2002 FFB-Reprint Nr. 26

Merz, J. und M. Zwick, Verteilungswirkungen der Steuerreform 2000/2005 im Vergleich zum ,Karlsruher Entwurf' - Auswirkungen auf die Einkommensverteilung bei Selbständigen (Freie Berufe, Unternehmer) und abhängig Beschäftigte, in: Wirtschaft und Statistik, 8/2002, S. 729-740, 2002, FFB-Reprint Nr. 25, Fachbereich Wirtschafts- und Sozialwissenschaften, Universität Lüneburg, Lüneburg. FFB-Reprint Nr. 25 
Merz, J., 2002, Time and Economic Well-Being - A Panel Analysis of Desired versus Actual Working Hours, in: Review of Income and Wealth, Series 48, No. 3, p. 317-346, FFB-Reprint Nr. 24, Department of Economics and Social Sciences, University of Lüneburg, Lüneburg.

FFB-Reprint Nr. 24

Schatz, Ch., Kortmann, K. und J. Merz, 2002, Künftige Alterseinkommen - Eine Mikrosimulationsstudie zur Entwicklung der Renten und Altersvorsorge in Deutschland (AVID’96), in: Schmollers Jahrbuch, Journal of Applied Social Sciences, Zeitschrift für Wirtschafts- und Sozialwissenschaften, 122. Jahrgang, Heft 2, S. 227-260, FFB-Reprint Nr. 23, Fachbereich Wirtschafts- und Sozialwissenschaften, Universität Lüneburg, Lüneburg.

FFB-Reprint Nr. 23

Merz, J. und M. Zwick, 2001, Über die Analyse hoher Einkommen mit der Einkommensteuerstatistik - Eine methodische Erläuterung zum Gutachten „Hohe Einkommen, ihre Struktur und Verteilung“ zum ersten Armuts- und Reichtumsbericht der Bundesregierung, in: Wirtschaft und Statistik 7/2001, S. 513-523, FFB-Reprint Nr. 22, Fachbereich Wirtschafts- und Sozialwissenschaften, Universität Lüneburg, Lüneburg.

FFB-Reprint Nr. 22

Merz, J., 2001, Was fehlt in der EVS? Eine Verknüpfung mit der Einkommensteuerstatistik für die Verteilungsanalyse hoher Einkommen, in: Becker, I., Ott, N. und G. Rolf (Hrsg.), Soziale Sicherung in einer dynamischen Gesellschaft, S. 278-300, Campus Verlag, Frankfurt/New York, FFB-Reprint Nr. 21, Fachbereich Wirtschafts- und Sozialwissenschaften, Universität Lüneburg, Lüneburg.

FFB-Reprint Nr. 21

Merz, J., 2001, 10 Jahre Forschungsinstitut Freie Berufe (FFB) der Universität Lüneburg - Forschungsschwerpunkte und Perspektiven, in: Bundesverband der Freien Berufe (Hrsg.), Jahrbuch 2000/2001, der freie beruf, S. 158-174, Bonn, FFB-Reprint Nr. 20, Fachbereich Wirtschafts- und Sozialwissenschaften, Universität Lüneburg, Lüneburg.

FFB-Reprint Nr. 20

Merz, J., 2000, The Distribution of Income of Self-employed, Entrepreneurs and Professions as Revealed from Micro Income Tax Statistics in Germany, in: Hauser, R. and I. Becker (eds.), The Personal Distribution of Income in an International Perspective, S. 99-128, Springer Verlag, Heidelberg, FFB-Reprint Nr.19, Department of Economics and Social Sciences, University of Lüneburg, Lüneburg. FFB-Reprint Nr. 19

Merz, J., 1998, Privatisierung, Deregulierung und staatlich gebundene Freie Berufe - Einige ökonomische Aspekte, in: Herrmann, H. und J. Backhaus (Hrsg.), Staatlich gebundene Freiberufe im Wandel, S. 67-114, FFB-Schriften Nr. 9, NOMOS Verlagsgesellschaft, Baden-Baden, FFB-Reprint Nr. 18, Fachbereich Wirtschafts- und Sozialwissenschaften, Universität Lüneburg, Lüneburg.

FFB-Reprint Nr. 18

Merz. J., 1997, Die Freien Berufe - Laudatio zur Verleihung der Ehrendoktorwürde des Fachbereiches Wirtschafts- und Sozialwissenschaften der Universität Lüneburg an Prof. J. F. Volrad Deneke, in: Bundesverband der Freien Berufe (Hrsg.), Jahrbuch 1997, der freie beruf, S. 133-151, Bonn, FFB-Reprint Nr. 17, Fachbereich Wirtschafts- und Sozialwissenschaften, Universität Lüneburg, Lüneburg. FFB-Reprint Nr. 17

de Kam, C. A., de Haan, J., Giles, C., Manresa, A., Berenguer, E., Calonge, S., Merz, J. and K. Venkatarama, 1996, Who pays the taxes? The Distribution of Effective Tax Burdens in Four EU Countries, in: ec Tax Review, p. 175-188, FFB-Reprint No. 16, Department of Economics and Social Sciences, University of Lüneburg, Lüneburg.

FFB-Reprint Nr. 16

Merz, J., 1996, MICSIM - A PC Microsimulation Model for Research and Teaching: A Brief View on its' Concept and Program Developments, SoftStat '95 - Advances in Statistical Software 5, in: Faulbaum, F. and W. Bandilla (eds.), Stuttgart, p. 433-442, FFB-Reprint No. 15, Department of Economics and Social Sciences, University of Lüneburg, Lüneburg.

FFB-Reprint Nr. 15

Burkhauser, R. V., Smeeding, T. M. and J. Merz, 1996, Relative Inequality and Poverty in Germany and the United States using Alternative Equivalence Scales, in: Review of Income and Wealth, Series 42, No. 4, p. 381-400, FFB-Reprint No. 14, Department of Economics and Social Sciences, University of Lüneburg, Lüneburg.

FFB-Reprint Nr. 14

Merz, J. 1996, Schattenwirtschaft und Arbeitsplatzbeschaffung, in: Sadowski, D. und K. Pull (Hrsg.), Vorschläge jenseits der Lohnpolitik, S. 266-294, Frankfurt/New York, FFB-Reprint Nr. 13, Fachbereich Wirtschafts- und Sozialwissenschaften, Universität Lüneburg, Lüneburg.

FFB-Reprint Nr. 13

Merz, J., 1996, MICSIM - Concept, Developments and Applications of a PC Microsimulation Model for Research and Teaching, in: Troitzsch, K. G., Mueller, U. Gilbert, G. N. and J. E. Doran (eds.), Social Sci- 
ence Microsimulation, p. 33-65, Berlin/New York, FFB-Reprint No. 12, Department of Economics and Social Sciences, University of Lüneburg, Lüneburg. FFB-Reprint Nr. 12

Merz, J., 1996, Market and Non-Market Labour Supply and the Impact of the Recent German Tax Reform Incorporating Behavioural Response, in: Harding, A. (ed.), Microsimulation and Public Policy, p. 177202, Amsterdam/Tokyo, FFB-Reprint No. 11, Department of Economics and Social Sciences, University of Lüneburg, Lüneburg.

FFB-Reprint Nr. 11

Merz, J. und R. Lang, 1996, Alles eine Frage der Zeit!? - Bericht über ein FFB-Forschungsprojekt zum Thema 'Zeitverwendung in Erwerbstätigkeit und Haushaltsproduktion - Dynamische Mikroanalysen mit Paneldaten', in: Uni Lüneburg - 50 Jahre Hochschule in Lüneburg, Jubiläums-Sonderausgabe, Heft 19/Mai 1996, S. 51-55, FFB-Reprint Nr. 10, Fachbereich Wirtschafts- und Sozialwissenschaften, Universität Lüneburg, Lüneburg.

FFB-Reprint Nr. 10

Merz, J. und D. Kirsten, 1996, Freie Berufe im Mikrozensus - Struktur, Einkommen und Einkommensverteilung in den neuen und alten Bundesländern, in: BFB Bundesverband der Freien Berufe (Hrsg.), Jahrbuch 1996, S. 40-79, Bonn, FFB-Reprint Nr. 9, Fachbereich Wirtschafts- und Sozialwissenschaften, Universität Lüneburg, Lüneburg.

FFB-Reprint Nr. 9

Deneke, J. F. V., 1995, Freie Berufe - Gestern, Heute, Morgen, in: BFB Bundesverband der Freien Berufe (Hrsg.), Jahrbuch 1995, S. 57-72, Bonn, FFB-Reprint Nr. 8, Fachbereich Wirtschafts- und Sozialwissenschaften, Universität Lüneburg, Lüneburg.

FFB-Reprint Nr. 8

Merz, J. and J. Faik, 1995, Equivalence Scales Based on Revealed Preference Consumption Expenditures - The Case of Germany, in: Jahrbücher für Nationalökonomie und Statistik, Vol. 214, No. 4, p. 425-447, Stuttgart, FFB-Reprint No. 7, Department of Economics and Social Sciences, University of Lüneburg, Lüneburg.

FFB-Reprint Nr. 7

Merz, J., 1993, Statistik und Freie Berufe im Rahmen einer empirischen Wirtschafts- und Sozialforschung, in: BFB Bundesverband der Freien Berufe (Hrsg.), Jahrbuch 1993, S. 31-78, Bonn, FFB-Reprint Nr. 6, Fachbereich Wirtschafts- und Sozialwissenschaften, Universität Lüneburg, Lüneburg. FFB-Reprint Nr. 6

Merz, J., 1993, Wandel in den Freien Berufen - Zum Forschungsbeitrag des Forschungsinstituts Freie Berufe (FFB) der Universität Lüneburg, in: BFB Bundesverband der Freien Berufe (Hrsg.), Jahrbuch 1993, S. 164-173, Bonn, FFB-Reprint Nr. 5, Fachbereich Wirtschafts- und Sozialwissenschaften, Universität Lüneburg, Lüneburg.

FFB-Reprint Nr. 5

Merz, J. and K. G. Wolff, 1993, The Shadow Economy: Illicit Work and Household Production: A Microanalysis of West Germany, in: Review of Income and Wealth, Vol. 39, No. 2, p. 177-194, FFB-Reprint No. 4, Department of Economics and Social Sciences, University of Lüneburg, Lüneburg. FFB-Reprint Nr. 4

Trautwein, H.-M. und A. Rönnau, 1993, Self-Regulation of the Medical Profession in Germany: A Survey, in: Faure, M., Finsinger, J., Siegers, J. und R. van den Bergh (eds.), Regulation of Profession, p. 249-305, ISBN 90-6215-334-8, MAKLU, Antwerpen, FFB-Reprint No. 3, Department of Economics and Social Sciences, University of Lüneburg, Lüneburg.

FFB-Reprint Nr. 3

Herrmann, H., 1993, Regulation of Attorneys in Germany: Legal Framework and Actual Tendencies of Deregulation, in: Faure, M., Finsinger, J., Siegers, J. und R. van den Bergh (eds.), Regulation of Profession, p. 225-245, ISBN 90-6215-334-8, MAKLU, Antwerpen, FFB-Reprint No. 2, Department of Economics and Social Sciences, University of Lüneburg, Lüneburg.

FFB-Reprint Nr. 2

Merz, J., 1991, Microsimulation - A Survey of Principles, Developments and Applications, in: International Journal of Forecasting 7, p. 77-104, ISBN 0169-2070-91, North-Holland, Amsterdam, FFB-Reprint Nr. 1, Department of Economics and Social Sciences, University of Lüneburg, Lüneburg.

FFB-Reprint Nr. 1

\section{$5 \quad$ FFB-Diskussionspapiere, ISSN 0942-2595}

Merz, J., Böhm, P., Hanglberger, D., Stolze, H. und R. Rucha, 2007, Wann werden Serviceleistungen nachgefragt? Eine Wirkungsanalyse anhand des neuen FFB-Mikrosimulationsmodells ServSim, FFBDiskussionspapier Nr. 70, Fakultät II, Wirtschafts-, Verhaltens- und Rechtswissenschaften, Universität Lüneburg, Lüneburg.

FFB-DP Nr. 70 
Stolze H., 2007, Die Nachfrage nach Gesundheitsleistungen - Ergebnisse der FFB-Patientenumfrage, FFBDiskussionspapier Nr. 69, Fakultät II, Wirtschafts-, Verhaltens- und Rechtswissenschaften, Universität Lüneburg, Lüneburg.

FFB-DP Nr. 69

Burgert, D., 2006, Betriebliche Weiterbildung und Verbleib Älterer im Betrieb, FFB-Diskussionspapier Nr. 68, Fakultät II, Wirtschafts-, Verhaltens- und Rechtswissenschaften, Universität Lüneburg, Lüneburg.

FFB-DP Nr. 68

Merz, J., 2006, Polarisierung der Einkommen von Selbständigen? - Zur Dynamik der Einkommensverteilung von Freiberuflern und Unternehmern, FFB-Diskussionspapier Nr. 67, Fakultät II, Wirtschafts-, Verhaltens- und Rechtswissenschaften, Universität Lüneburg, Lüneburg.

FFB-DP Nr. 67

Paic, P., 2006, Informationelle Zugänge für die empirische Untersuchung freiberuflicher Existenzgründungen, FFB-Diskussionspapier Nr. 66, Fakultät II, Wirtschafts-, Verhaltens- und Rechtswissenschaften, Universität Lüneburg, Lüneburg.

FFB-DP Nr. 66

Merz, J. und M. Zwick, 2006, Einkommensanalysen mit Steuerdaten - Mikroanalysen zu hohen Einkommen und Selbständigkeit und Mikrosimulation zu Politikalternativen der Einkommen-, Körperschaft- und Gewerbesteuer, FFB-Diskussionspapier Nr. 65, Fakultät II, Wirtschafts-, Verhaltens- und Rechtswissenschaften, Universität Lüneburg, Lüneburg.

FFB-DP Nr. 65

Merz, J., Stolze, H. und M. Zwick, 2006, Wirkungen alternativer Steuerreformmodelle auf die Einkommensverteilung von Freien und anderen Berufen, FFB-Diskussionspapier Nr. 64, Fakultät II, Wirtschafts-, Verhaltens- und Rechtswissenschaften, Universität Lüneburg, Lüneburg.

FFB-DP Nr. 64

Zwick, M., 2006, Gemeindefinanzreform und Freie Berufe, FFB-Diskussionspapier Nr. 63, Fakultät II, Wirtschafts-, Verhaltens- und Rechtswissenschaften, Universität Lüneburg, Lüneburg.

FFB-DP Nr. 63

Burgert, D., 2006, Kündigungsschutz - Ein Hindernis für das Beschäftigungswachstum? FFBDiskussionspapier Nr. 62, Fakultät II, Wirtschafts-, Verhaltens- und Rechtswissenschaften, Universität Lüneburg, Lüneburg.

FFB-DP Nr. 62

Paic, P., 2006, Existenzgründung und Einkommen Freier Berufe - Ergebnisse aus dem Sozio-ökonomischen Panel und der FFB-Onlineumfrage Freie Berufe, FFB-Diskussionspapier Nr. 61, Fakultät II, Wirtschafts-, Verhaltens- und Rechtswissenschaften, Universität Lüneburg, Lüneburg.

FFB-DP Nr. 61

Merz, J. und P. Böhm, 2006, Arbeitszeit und Einkommen - Neue Ergebnisse aus der deutschen Zeitbudgeterhebung, FFB-Diskussionspapier Nr. 60, Fakultät II, Wirtschafts-, Verhaltens- und Rechtswissenschaften, Universität Lüneburg, Lüneburg.

FFB-DP Nr. 60

Merz, J., 2006, Hohe und niedrige Einkommen - Neue Ergebnisse zu Freien und anderen Berufen aus dem Armuts- und Reichtumsbericht der Bundesregierung, FFB-Diskussionspapier Nr. 59, Fakultät II, Wirtschafts-, Verhaltens- und Rechtswissenschaften, Universität Lüneburg, Lüneburg.

FFB-DP Nr. 59

Merz, J., Daniel Vorgrimler und Markus Zwick, 2006, De facto Anonymised Microdata File on Income Tax Statistics 1998, FFB-Diskussionspapier Nr. 58, Fakultät II, Wirtschafts-, Verhaltens- und Rechtswissenschaften, Universität Lüneburg, Lüneburg.

FFB-DP Nr. 58

Merz, J. and L. Osberg, 2006, Keeping in Touch - A Benefit of Public Holidays. FFB-Diskussionspapier Nr. 57, Fakultät II, Wirtschafts-, Verhaltens- und Rechtswissenschaften, Universität Lüneburg, Lüneburg

FFB-DP Nr. 57

Merz, J. und P. Paic, 2006, Start-up success of freelancers - New microeconometric evidence from the German Socio-Economic Panel. FFB-Diskussionspapier Nr. 56, Fakultät II, Wirtschafts-, Verhaltens- und Rechtswissenschaften, Universität Lüneburg, Lüneburg.

FFB-DP Nr. 56

Merz, J. und P. Paic, 2006, Erfolgsfaktoren freiberuflicher Existenzgründung - Neue mikroökonometrische Ergebnisse mit Daten des Sozio-ökonomischen Panels, FFB-Diskussionspapier Nr. 55, Fakultät II, Wirtschafts-, Verhaltens- und Rechtswissenschaften, Universität Lüneburg, Lüneburg.

FFB-DP Nr. 55 
Merz, J. und H., Stolze, 2006, Representative Time Use Data and Calibration of the American Time Use Studies 1965-1999, FFB-Diskussionspapier Nr. 54, Fakultät II, Wirtschafts-, Verhaltens- und Rechtswissenschaften, Universität Lüneburg, Lüneburg.

FFB-DP Nr. 54

Paic, P. und H. Brand, 2005, Die Freien Berufe im Sozio-ökonomischen Panel - Systematische Berichtigung der kritischen Wechsel innerhalb der Selbständigengruppe, FFB-Diskussionspapier Nr. 53, Fachbereich Wirtschafts- und Sozialwissenschaften, Universität Lüneburg, Lüneburg.

FFB-DP Nr. 53

Merz, J. und P. Paic, 2005, Zum Einkommen der Freien Berufe - Eine Ordered Probit-Analyse ihrer Determinanten auf Basis der FFB-Onlineumfrage, FFB-Diskussionspapier Nr. 52, Fachbereich Wirtschafts- und Sozialwissenschaften, Universität Lüneburg, Lüneburg.

FFB-DP Nr. 52

Burgert, D., 2005, Schwellenwerte im deutschen Kündigungsschutzrecht - Ein Beschäftigungshindernis für kleine Unternehmen?, FFB-Diskussionspapier Nr. 51, Fachbereich Wirtschafts- und Sozialwissenschaften, Universität Lüneburg, Lüneburg.

FFB-DP Nr. 51

Merz, J., Böhm, P. und D. Burgert, 2005, Arbeitszeitarrangements und Einkommensverteilung - Ein Treatment Effects Ansatz der Einkommensschätzung für Unternehmer, Freiberufler und abhängig Beschäftigte, FFB-Diskussionspapier Nr. 50, Fachbereich Wirtschafts- und Sozialwissenschaften, Universität Lüneburg, Lüneburg.

FFB-DP Nr. 50

Burgert, D., 2005, The Impact of German Job Protection Legislation on Job Creation in Small Establishments An Application of the Regression Discontinuity Design, FFB-Diskussionspapier Nr. 49, Fachbereich Wirtschafts- und Sozialwissenschaften, Universität Lüneburg, Lüneburg.

FFB-DP Nr. 49

Merz, J. und D. Burgert, 2005, Arbeitszeitarrangements - Neue Ergebnisse aus der nationalen Zeitbudgeterhebung 2001/02 im Zeitvergleich, FFB-Diskussionspapier Nr. 48, Fachbereich Wirtschafts- und Sozialwissenschaften, Universität Lüneburg, Lüneburg.

FFB-DP Nr. 48

Merz, J., Böhm, P. und D. Burgert, 2005, Timing, Fragmentation of Work and Income Inequality - An Earnings Treatment Effects Approach, FFB-Diskussionspapier Nr. 47, Fachbereich Wirtschafts- und Sozialwissenschaften, Universität Lüneburg, Lüneburg.

FFB-DP Nr. 47

Merz, J. und P. Paic, 2004, Existenzgründungen von Freiberuflern und Unternehmer - Eine Mikroanalyse mit dem Sozio-ökonomischen Panel FFB-Diskussionspapier Nr. 46, Fachbereich Wirtschafts- und Sozialwissenschaften, Universität Lüneburg, Lüneburg.

FFB-DP Nr. 46

Merz, J. und D. Burgert, 2004, Wer arbeitet wann? Arbeitszeitarrangements von Selbständigen und abhängig Beschäftigten - Eine mikroökonometrische Analyse deutscher Zeitbudgetdaten, FFB-Diskussionspapier Nr. 45, Fachbereich Wirtschafts- und Sozialwissenschaften, Universität Lüneburg, Lüneburg.FFB-DP Nr. 45

Hirschel, D. und J. Merz, 2004, Was erklärt hohe Arbeitseinkommen der Selbständigen - Eine Mikroanalyse mit Daten des Sozio-ökonomsichen Panels, FFB-Diskussionspapier Nr. 44, Fachbereich Wirtschafts- und Sozialwissenschaften, Universität Lüneburg, Lüneburg.

FFB-DP Nr. 44

Ackermann, D., Merz, J. und H. Stolze, 2004, Erfolg und Erfolgsfaktoren freiberuflich tätiger Ärzte - Ergebnisse der FFB-Ärztebefragung für Niedersachsen, FFB-Diskussionspapier Nr. 43, Fachbereich Wirtschaftsund Sozialwissenschaften, Universität Lüneburg, Lüneburg.

FFB-DP Nr. 43

Hirschel, D. 2003, Do high incomes reflect individual performance? The determinants of high incomes in Germany, FFB-Diskussionspapier Nr. 42, Department of Economics and Social Sciences, University of Lüneburg, Lüneburg.

FFB-DP Nr. 42

Merz, J., and D. Burgert, 2003, Working Hour Arrangements and Working Hours - A Microeconometric Analysis Based on German Time Diary Data, FFB-Diskussionspapier Nr. 41, Department of Economics and Social Sciences, University of Lüneburg, Lüneburg.

FFB-DP Nr. 41

Merz, J. und M. Zwick, 2002, Hohe Einkommen: Eine Verteilungsanalyse für Freie Berufe, Unternehmer und abhängig Beschäftigte, Eine Mikroanalyse auf der Basis der Einkommensteuerstatistik, FFBDiskussionspapier Nr. 40, Fachbereich Wirtschafts- und Sozialwissenschaften, Universität Lüneburg, Lüneburg.

FFB-DP Nr. 40 
Merz, J. and D. Hirschel, 2003, The distribution and re-distribution of income of self-employed as freelancers and entrepreneurs in Europe, FFB-Diskussionspapier Nr. 39, Department of Economics and Social Sciences, University of Lüneburg, Lüneburg.

FFB-DP Nr. 39

Stolze, H. 2002, Datenbankbankgestützte Internetpräsenzen - Entwicklung und Realisation am Beispiel der Homepage des Forschungsinstituts Freie Berufe (FFB) der Universität Lüneburg http://ffb.unilueneburg.de, FFB-Diskussionspapier Nr. 38, Fachbereich Wirtschafts- und Sozialwissenschaften, Universität Lüneburg, Lüneburg.

FFB-DP Nr. 38

Merz, J., 2002, Zur Kumulation von Haushaltsstichproben, FFB-Diskussionspapier Nr. 37, Fachbereich Wirtschafts- und Sozialwissenschaften, Universität Lüneburg, Lüneburg.

FFB-DP Nr. 37

Merz, J., 2002, Reichtum in Deutschland: Hohe Einkommen, ihre Struktur und Verteilung - Eine Mikroanalyse mit der Einkommensteuerstatistik für Selbständige und abhängig Beschäftigte, FFB-Diskussionspapier Nr. 36, Fachbereich Wirtschafts- und Sozialwissenschaften, Universität Lüneburg, Lüneburg.FFB-DP Nr. 36

Merz, J. und M. Zwick, 2002, Verteilungswirkungen der Steuerreform 2000/2005 im Vergleich zum ,Karlsruher Entwurf Auswirkungen auf die Einkommensverteilung bei Selbständigen (Freie Berufe, Unternehmer und abhängig Beschäftigte), FFB-Diskussionspapier Nr. 35, Fachbereich Wirtschafts- und Sozialwissenschaften, Universität Lüneburg, Lüneburg.

FFB-DP Nr. 35

Merz, J., Stolze, H. und M. Zwick, 2002, Professions, entrepreneurs, employees and the new German tax (cut) reform 2000 - A MICSIM microsimulation analysis of distributional impacts, FFB-Diskussionspapier Nr. 34, Department of Economics and Social Sciences, University of Lüneburg, Lüneburg. FFB-DP Nr. 34

Forschungsinstitut Freie Berufe, 2002, Freie Berufe im Wandel der Märkte - 10 Jahre Forschungsinstitut Freie Berufe (FFB) der Universität Lüneburg, Empfang am 4. November 1999 im Rathaus zu Lüneburg, FFBDiskussionspapier Nr. 33, Fachbereich Wirtschafts- und Sozialwissenschaften, Universität Lüneburg, Lüneburg.

FFB-DP Nr. 33

Merz, J., 2002, Time Use Research and Time Use Data - Actual Topics and New Frontiers, FFB-Discussion Paper No. 32, Department of Economics and Social Sciences, University of Lüneburg, Lüneburg.

FFB-DP Nr. 32

Merz, J., 2001, Freie Berufe im Wandel der Arbeitsmärkte, FFB-Diskussionspapier Nr. 31, Fachbereich Wirtschafts-und Sozialwissenschaften, Universität Lüneburg, Lüneburg.

FFB-DP Nr. 31

Merz, J., 2001, Was fehlt in der EVS? Eine Verteilungsanalyse hoher Einkommen mit der verknüpften Einkommensteuerstatistik für Selbständige und abhängig Beschäftigte, FFB-Diskussionspapier Nr. 30, Fachbereich Wirtschafts- und Sozialwissenschaften, Universität Lüneburg, Lüneburg. $\quad$ FFB-DP Nr. 30

Merz, J., 2001, Informationsfeld Zeitverwendung - Expertise für die Kommission zur Verbesserung der informationellen Infrastruktur zwischen Wissenschaft und Statistik, FFB-Diskussionspapier Nr. 29, Fachbereich Wirtschafts- und Sozialwissenschaften, Universität Lüneburg, Lüneburg.

FFB-DP Nr. 29

Schatz, C. und J. Merz, 2000, Die Rentenreform in der Diskussion - Ein Mikrosimulationsmodell für die Altersvorsorge in Deutschland (AVID-PTO), FFB-Diskussionspapier Nr. 28, Fachbereich Wirtschafts- und Sozialwissenschaften, Universität Lüneburg, Lüneburg.

FFB-DP Nr. 28

Merz, J., 2000, The Distribution of Income of Self-employed, Entrepreneurs and Professions as Revealed from Micro Income Tax Statistics in Germany, FFB-Discussion Paper No. 27, Department of Economics and Social Sciences, University of Lüneburg, Lüneburg.

FFB-DP Nr. 27

Merz, J., Loest, O. und A. Simon, 1999, Existenzgründung - Wie werde ich selbständig, wie werde ich Freiberufler? Ein Leitfaden, FFB-Diskussionspapier Nr. 26, Fachbereich Wirtschafts- und Sozialwissenschaften, Universität Lüneburg, Lüneburg.

FFB-DP Nr. 26

Merz, J. und D. Kirsten, 1998, Extended Income Inequality and Poverty Dynamics of Labour Market and Valued Household Acitivities - A Ten Years Panelanalysis for Professions, Entrepreneurs and Employees in Germany, FFB-Discussion Paper No. 25, Department of Economics and Social Sciences, University of Lüneburg, Lüneburg.

FFB-DP Nr. 25 
Merz, J., Quiel, T. und K. Venkatarama, 1998, Wer bezahlt die Steuern? - Eine Untersuchung der Steuerbelastung und der Einkommenssituation für Freie und andere Berufe, FFB-Diskussionspapier Nr. 24, Fachbereich Wirtschafts- und Sozialwissenschaften, Universität Lüneburg, Lüneburg.

FFB-DP Nr. 24

Merz, J. und R. Lang, 1997, Preferred vs. Actual Working Hours - A Ten Paneleconometric Analysis for Professions, Entrepreneurs and Employees in Germany, FFB-Discussion Paper No. 23, Department of Economics and Social Sciences, University of Lüneburg, Lüneburg.

FFB-DP Nr. 23

Merz, J., 1997, Privatisierung und Deregulierung und Freie und staatlich gebundene Freie Berufe - Einige ökonomische Aspekte, FFB-Diskussionspapier Nr. 22, Fachbereich Wirtschafts- und Sozialwissenschaften, Universität Lüneburg, Lüneburg.

FFB-DP Nr. 22

de Kam, C. A., de Haan, J., Giles, C., Manresa, A., Berenguer, E., Calonge, S., Merz, J. and K. Venkatarama, 1996, The Distribution of Effetcive Tax Burdens in Four EU Countries, FFB-Discussion Paper No. 21, Department of Economics and Social Sciences, University of Lüneburg, Lüneburg.

FFB-DP Nr. 21

Deneke, J. F. V., 1996, Freie Berufe und Mittelstand - Festrede zur Verleihung der Ehrendoktorwürde, FFBDiskussionspapier Nr. 20, Fachbereich Wirtschafts- und Sozialwissenschaften, Universität Lüneburg, Lüneburg.

FFB-DP Nr. 20

Merz, J., 1996, Die Freien Berufe - Laudatio zur Verleihung der Ehrendokotorwürde des Fachbereiches Wirtschafts- und Sozialwissenschaften der Universität Lüneburg an Prof. J. F. Volrad Deneke, FFBDiskussionspapier Nr. 19, Fachbereich Wirtschafts- und Sozialwissenschaften, Universität Lüneburg, Lüneburg.

FFB-DP Nr. 19

de Kam, C. A., de Haan, J., Giles, C., Manresa, A., Berenguer, E., Calonge, S. and J. Merz, 1996, Who pays the taxes?, FFB-Discussion Paper No. 18, Department of Economics and Social Sciences, University of Lüneburg, Lüneburg.

FFB-DP Nr. 18

Merz, J., 1996, Schattenwirtschaft und ihre Bedeutung für den Arbeitsmarkt, FFB-Diskussionspapier Nr. 17, Fachbereich Wirtschafts- und Sozialwissenschaften, Universität Lüneburg, Lüneburg.

FFB-DP Nr. 17

Merz, J. und D. Kirsten, 1995, Freie Berufe im Mikrozensus II - Einkommen und Einkommensverteilung anhand der ersten Ergebnisse für die neuen und alten Bundesländer 1991, FFB-Diskussionspapier Nr. 16, Fachbereich Wirtschafts- und Sozialwissenschaften, Universität Lüneburg, Lüneburg. $\quad$ FFB-DP Nr. 16

Merz, J. und D. Kirsten, 1995, Freie Berufe im Mikrozensus I - Struktur und quantitative Bedeutung anhand der ersten Ergebnisse für die neuen und alten Bundesländer 1991, FFB-Diskussionspapier Nr. 15, Fachbereich Wirtschafts- und Sozialwissenschaften, Universität Lüneburg, Lüneburg.

FFB-DP Nr. 15

Merz, J., 1995, MICSIM - Concept, Developments and Applications of a PC-Microsimulation Model for Research and Teaching, FFB-Discussion Paper No. 14, Department of Economics and Social Sciences, University of Lüneburg, Lüneburg.

FFB-DP Nr. 14

Rönnau, A., 1995, Freie Berufe in der DDR, der Bundesrepublik Deutschland und im wiedervereinten Deutschland: Auswertungen von Berufstätigenerhebung und Arbeitsstättenzählung, FFB-Diskussionspapier Nr. 13, Fachbereich Wirtschafts- und Sozialwissenschaften, Universität Lüneburg, Lüneburg. FFB-DP Nr. 13

Burkhauser, R. V., Smeeding, T. M. and J. Merz, 1994, Relative Inequality and Poverty in Germany and the United States Using Alternative Equivalence Scales, FFB-Discussion Paper No. 12, Department of Economics and Social Sciences, University of Lüneburg, Lüneburg.

FFB-DP Nr. 12

Widmaier, U., Niggemann, H. and J. Merz, 1994, What makes the Difference between Unsuccessful and Successful Firms in the German Mechanical Engineering Industry? A Microsimulation Approach Using Data from the NIFA-Panel, FFB-Discussion Paper No. 11, Department of Economics and Social Sciences, University of Lüneburg, Lüneburg.

FFB-DP Nr. 11

Merz, J., 1994, Microdata Adjustment by the Minimum Information Loss Principle, FFB-Discussion Paper No. 10, Department of Economics and Social Sciences, University of Lüneburg, Lüneburg. FFB-DP Nr. 10

Merz, J., 1994, Microsimulation - A Survey of Methods and Applications for Analyzing Economic and Social Policy, FFB-Discussion Paper No. 9, Department of Economics and Social Sciences, University of Lüneburg, Lüneburg.

FFB-DP Nr. 9 
Merz, J., Garner, T., Smeeding, T. M., Faik, J. and D. Johnson, 1994, Two Scales, One Methodology - Expenditure Based Equivalence Scales for the United States and Germany, FFB-Discussion Paper No. 8, Department of Economics and Social Sciences, University of Lüneburg, Lüneburg.

FFB-DP Nr. 8

Krickhahn, T., 1993, Lobbyismus und Mittelstand: Zur Identifikation der Interessenverbände des Mittelstands in der Bundesrepublik Deutschland, FFB-Diskussionspapier Nr. 7, Fachbereich Wirtschafts- und Sozialwissenschaften, Universität Lüneburg, Lüneburg.

FFB-DP Nr. 7

Merz, J., 1993, Market and Non-Market Labor Supply and Recent German Tax Reform Impacts - Behavioral Response in a Combined Dynamic and Static Microsimulation Model, FFB-Discussion Paper No. 6, Department of Economics and Social Sciences, University of Lüneburg, Lüneburg.

FFB-DP Nr. 6

Merz, J., 1993, Microsimulation as an Instrument to Evaluate Economic and Social Programmes, FFBDiscussion Paper No. 5, Department of Economics and Social Sciences, University of Lüneburg, Lüneburg.

FFB-DP Nr. 5

Merz, J., 1993, Statistik und Freie Berufe im Rahmen einer empirischen Wirtschafts- und Sozialforschung, Antrittsvorlesung im Fachbereich Wirtschafts- und Sozialwissenschaften der Universität Lüneburg, FFBDiskussionspapier Nr. 4, Fachbereich Wirtschafts- und Sozialwissenschaften, Universität Lüneburg, Lüneburg.

FFB-DP Nr. 4

Merz, J. and J. Faik, 1992, Equivalence Scales Based on Revealed Preference Consumption Expenditure Microdata - The Case of West Germany, FFB-Discussion Paper No. 3, Department of Economics and Social Sciences, University of Lüneburg, Lüneburg.

FFB-DP Nr. 3

Merz, J., 1992, Time Use Dynamics in Paid Work and Household Activities of Married Women - A Panel Analysis with Household Information and Regional Labour Demand, FFB-Discussion Paper No. 2, Department of Economics and Social Sciences, University of Lüneburg, Lüneburg.

FFB-DP Nr. 2

Forschungsinstitut Freie Berufe, 1992, Festliche Einweihung des Forschungsinstituts Freie Berufe am 16. Dezember 1991 im Rathaus zu Lüneburg, FFB-Diskussionspapier Nr. 1, Fachbereich Wirtschafts- und Sozialwissenschaften, Universität Lüneburg, Lüneburg.

FFB-DP Nr. 1

\section{FFB-Dokumentationen, ISSN 1615-0376}

Merz, J., Hillebrecht, H., Rucha, R., Schlüter, H. und H. Stolze, 2006, e-learning für das Fach Statistik - Ein Pilotprojekt an der Universität Lüneburg, FFB-Dokumentation Nr. 14, Fachbereich Wirtschafts- und Sozialwissenschaften, Universität Lüneburg, Lüneburg.

FFB-Dok. Nr. 14

Merz, J. und H. Stolze, 2006, Adjust for Windows Version 1.1 - A Software Package to Achieve Representative Microdata by the Minimum Information Loss Principle, Program Manual, FFB-Dokumentation Nr. 13, Fakultät Wirtschafts-, Verhaltens- und Rechtswissenschaften, Universität Lüneburg, Lüneburg

FFB-Dok. Nr. 13

Merz, J. und P. Paic, 2005, Die FFB-Onlineumfrage Freie Berufe - Beschreibung und Hochrechnung, FFBDokumentation Nr. 12, Fakultät Wirtschafts-, Verhaltens- und Rechtswissenschaften, Universität Lüneburg, Lüneburg

FFB-Dok. Nr. 12

Stolze, H., 2004, Der FFB-Server mit Microsoft Windows Server 2003, FFB-Dokumentation Nr. 11, Fachbereich Wirtschafts- und Sozialwissenschaften, Universität Lüneburg, Lüneburg.

FFB-Dok. Nr. 11

Stolze, H., 2004, Zur Repräsentativität der FFB-Ärzteumfrage - Neue Hochrechnungen für Niedersachsen, Nordrhein und Deutschland, FFB-Dokumentation Nr. 10, Fachbereich Wirtschafts- und Sozialwissenschaften, Universität Lüneburg, Lüneburg.

FFB-Dok. Nr. 10

Merz, J., Stolze, H. und S. Imme, 2001, ADJUST FOR WINDOWS - A Program Package to Adjust Microdata by the Minimum Information Loss Principle, Program-Manual, FFB-Dokumentation No. 9, Department of Economics and Social Sciences, University of Lüneburg, Lüneburg.

FFB-Dok. Nr. 9

Merz, J., Fink, F., Plönnigs, F. und T. Seewald, 1999, Forschungsnetz Zeitverwendung - Research Network on Time Use (RNTU), FFB-Dokumentation Nr. 8, Fachbereich Wirtschafts- und Sozialwissenschaften, Universität Lüneburg, Lüneburg.

FFB-Dok. Nr. 8 
Merz, J., 1997, Zeitverwendung in Erwerbstätigkeit und Haushaltsproduktion - Dynamische Mikroanalysen mit Paneldaten, DFG-Endbericht, FFB-Dokumentation Nr. 7, Fachbereich Wirtschafts- und Sozialwissenschaften, Universität Lüneburg, Lüneburg.

FFB-Dok. Nr. 7

Merz, J. und F. Plönnigs, 1997, DISTRI/MICSIM - A Softwaretool for Microsimulation Models and Analyses of Distribution, FFB-Dokumentation No. 6, Department of Economics and Social Sciences, University of Lüneburg, Lüneburg.

FFB-Dok. Nr. 6

Merz, J. und R. Lang, 1997, Neue Hochrechnung der Freien Berufe und Selbständigen im Sozio-ökonomischen Panel, FFB-Dokumentation Nr. 5, Fachbereich Wirtschafts- und Sozialwissenschaften, Universität Lüneburg, Lüneburg.

FFB-Dok. Nr. 5

Merz, J. und F. Plönnigs, 1995, Forschungsinstitut Freie Berufe - Datenschutz und Datensicherung, FFBDokumentation Nr. 4, Fachbereich Wirtschafts- und Sozialwissenschaften, Universität Lüneburg, Lüneburg.

FFB-Dok. Nr. 4

Merz, J., Hecker, M., Matusall, V. und H. Wiese, 1994, Forschungsinstitut Freie Berufe - EDV-Handbuch, FFB-Dokumentation Nr. 3, Fachbereich Wirtschafts- und Sozialwissenschaften, Universität Lüneburg, Lüneburg.

FFB-Dok. Nr. 3

Merz, J., 1993, Zeitverwendung in Erwerbstätigkeit und Haushaltsproduktion - Dynamische Mikroanalysen mit Paneldaten, DFG-Zwischenbericht für die erste Phase (1992-1993), FFB-Dokumentation Nr. 2, Fachbereich Wirtschafts- und Sozialwissenschaften, Universität Lüneburg, Lüneburg.

FFB-Dok. Nr. 2

Merz, J., 1993, ADJUST - Ein Programmpaket zur Hochrechnung von Mikrodaten nach dem Prinzip des minimalen Informationsverlustes, Programm-Handbuch, FFB-Dokumentation Nr. 1, Fachbereich Wirtschaftsund Sozialwissenschaften, Universität Lüneburg, Lüneburg.

FFB-Dok. Nr. 1

Available also in English as:

Merz, J., 1994, ADJUST - A Program Package to Adjust Microdata by the Minimum Information Loss Principle, Program-Manual, FFB-Dokumentation No. 1e, Department of Economics and Social Sciences, University of Lüneburg, Lüneburg.

FFB-Dok. Nr. 1e

\section{Sonstige Arbeitsberichte, ISSN 0175-7275}

Matusall, V., Kremers, H. und G. Behling, 1992, Umweltdatenbanken - vom Konzept zum Schema, Arbeitsbericht Nr. 112, Universität Lüneburg, Lüneburg. Mikrosimulation in der Steuerpolitik, 279 Seiten, Springer Verlag, ISBN 3-7908-0611-0, Berlin.

Preis: $€$ 6,-

Rönnau, A., 1989, Freie Berufe in Niedersachsen - Numerische und wirtschaftliche Entwicklung; Bedeutung als Arbeitgeber, Arbeitsbericht Nr. 60, Universität Lüneburg, Lüneburg. Mikrosimulation in der Steuerpolitik, 279 Seiten, Springer Verlag Berlin, ISBN 3-7908-0611-0, Berlin.

Preis: € 6,-

Sahner, H., 1989, Freie Berufe im Wandel, Arbeitsbericht Nr. 59, Universität Lüneburg, Lüneburg. Mikrosimulation in der Steuerpolitik, 279 Seiten, Springer Verlag, ISBN 3-7908-0611-0, Berlin.

Preis: $€$ 6,-

Sahner, H., 1988, Die Interessenverbände in der Bundesrepublik Deutschland - Ein Klassifikationssystem zu ihrer Erfassung, Arbeitsbericht Nr. 41, Universität Lüneburg, Lüneburg. Mikrosimulation in der Steuerpolitik, 279 Seiten, Springer Verlag, ISBN 3-7908-0611-0, Berlin.

Preis: € 6,-

FFB-Jahresberichte und FFB-Reprints werden auf Anfrage kostenlos zugesandt.

FFB-Diskussionspapiere und FFB-Dokumentationen können über unsere FFB-Homepage kostenlos heruntergeladen werden.

Die FFB-Bücher können sowohl bei uns als auch beim NOMOS Verlag bestellt werden. Sonstige Arbeitsberichte und Sonstige Bücher sind über den jeweiligen Autor zu beziehen.

http://ffb.uni-lueneburg.de 